\title{
Fixed Point Theorems for a New Class of Mappings in Modular Spaces Endowed with a Graph
}

\author{
Nour-eddine El Harmouchi $\left(\mathbb{D}\right.$, Karim Chaira $\mathbb{D}^{D}$, and El Miloudi Marhrani \\ Laboratory of Algebra, Analysis and Applications (L3A), Faculty of Sciences Ben M'Sik, Hassan II University of Casablanca, \\ Avenue Cdt Driss El Harti, B.P 7955 Sidi Othmane, Casablanca, Morocco \\ Correspondence should be addressed to El Miloudi Marhrani; marhrani@gmail.com
}

Received 12 January 2020; Revised 29 March 2020; Accepted 23 April 2020; Published 17 July 2020

Academic Editor: Patricia J. Y. Wong

Copyright (c) 2020 Nour-eddine El Harmouchi et al. This is an open access article distributed under the Creative Commons Attribution License, which permits unrestricted use, distribution, and reproduction in any medium, provided the original work is properly cited.

In this paper, we discuss a class of mappings more general than $\rho$-nonexpansive mapping defined on a modular space endowed with a graph. In our investigation, we prove the existence of fixed point results of these mappings. Then, we also introduce an iterative scheme for which proves the convergence to a fixed point of such mapping in a modular space with a graph.

\section{Introduction}

The abstract definition of modular spaces was introduced by Nakano [1] in 1950. Further, Musielak and Orlicz [2] redefined the modular spaces in such a way that a modular function space is seen as a vector space endowed with a modular function.

Fixed point theorems for monotone mappings in metric spaces endowed with partial ordering are considered firstly by Ran and Reurings [3] in 2004. They gave a generalization of Banach's contraction theorem in partially ordered metric spaces. Their results were extended by Neito and Rodríguez-López [4]. Following this line of research, fixed point theory rapidly developed in the framework of spaces equipped with partial ordering. Some related works are [5-7].

Graphs are an ordered structure that contains and generalizes partial ordering as a particular case. The use of graphs, in the study of fixed points, was introduced by Jachymski [8]. The main idea of his work is that the contraction inequality satisfied only for some connected vertices of a graph. Therefore, he generalized Banach's theorem in metric spaces with a graph. This work opened a new direction of research for the development of fixed point theory, in which many papers appeared [9-13].
In the last 10 years, many authors have considered the approach of Jachymski in the setting of modular spaces. Öztürk et al. [14] presented some fixed point results for mappings satisfying the integral contraction condition in a vector modular space equipped with a graph. Also, Alfuraidan [15] proved a generalization of Banach's theorem in modular metric spaces endowed with a graph. Afterwards, Alfuraidan examined in [16] the existence of fixed points for multivalued monotone $G$-contraction and $G$-nonexpansive mappings in modular function spaces.

Over the past few decades, several iterative methods have been proposed by many mathematicians to find and approximate fixed points of nonlinear mappings. Some references to iterative processes are [17-23].

In 2016, Ullah and Arshad [24] introduced a new iterative algorithm named AK iteration, defined by the following: for a given $x_{0} \in C$,

$$
\left\{\begin{array}{l}
x_{n+1}=T\left(y_{n}\right), \\
y_{n}=T\left(\left(1-\alpha_{n}\right) z_{n}+\alpha_{n} T\left(z_{n}\right)\right), \\
z_{n}=T\left(\left(1-\beta_{n}\right) x_{n}+\beta_{n} T\left(x_{n}\right)\right), \quad n \in \mathbb{N},
\end{array}\right.
$$

where $\left(\alpha_{n}\right)_{n}$ and $\left(\beta_{n}\right)_{n}$ are in $(0,1)$. According to Ullah and Arshad, this scheme converges to fixed points of contractions 
and also has a better converge rate than all previous processes.

Subsequently, many authors extended and studied the convergence of iterative methods for nonlinear maps in various spaces endowed with a graph.

In 2012, Aleomraninejad et al. [25] proved the convergence of certain iterative processes for $G$-contraction and $G$ -nonexpansive mapping acting on a graph. Alfuraidan [16] justified the existence and convergence for multivalued mapping in modular function spaces endowed with a graph. He also gave some results on the approximation of fixed points of Ćirić quasicontraction mappings in metric modular spaces equipped with a graph (see [15]).

In this paper, we start by introducing a new generalization of $\rho$-nonexpansive mappings. Using Mann's [20] iteration, we prove the existence of fixed points of this large class in modular spaces endowed with an oriented graph. Afterwards, we establish some convergence results for a new iterative process (see (55)) which can be considered an accelerated version of the $\mathrm{AK}$ iteration scheme.

\section{Preliminaries}

Throughout this work, $X$ stands for a linear vector space on the field $R$.

Definition 1 (see [26]). A function $\rho: X \longrightarrow[0,+\infty]$ is called a modular if the following holds:

(1) $\rho(x)=0$ if and only if $x=0$

(2) $\rho(-x)=\rho(x)$

(3) $\rho(\alpha x+(1-\alpha) y) \leq \rho(x)+\rho(y)$ for any $\alpha \in[0,1]$

for any $x$ and $y$ in $X$.

If (3) is replaced by $\left(3^{\prime}\right) \rho(\alpha x+(1-\alpha) y) \leq \alpha \rho(x)+(1-$ $\alpha) \rho(y)$ for any $\alpha \in[0,1]$ and $x$ and $y$ in $X$, then $\rho$ is called a convex modular.

A modular $\rho$ defines a corresponding modular space, i.e., the vector space $X_{\rho}$ given by

$$
X_{\rho}=\left\{x \in X: \lim _{\lambda \rightarrow 0} \rho(\lambda x)=0\right\} .
$$

Let $\rho$ be a convex modular, and the modular space $X_{\rho}$ is equipped with a norm called the Luxemburg norm, defined by

$$
\|x\|_{\rho}=\inf \left\{\lambda>0: \rho\left(\frac{x}{\lambda}\right) \leq 1\right\} .
$$

In the next definition, we give the basic definitions needed throughout this work.

Definition 2 (see [26]). Let $\rho$ be a modular defined on a vector space $X$.
(1) We say that a sequence $\left(x_{n}\right)_{n \in \mathbb{N}} \subset X_{\rho}$ is $\rho$-convergent to $x \in X_{\rho}$ if and only if $\rho\left(x_{n}-x\right)$ converges to 0 when $n$ goes to infinity. Note that the limit is unique

(2) A sequence $\left(x_{n}\right)_{n} \subset X_{\rho}$ is called $\rho$-Cauchy if $\rho\left(x_{n}-x_{m}\right) \longrightarrow 0$ as $n, m \longrightarrow+\infty$

(3) We say that $X_{\rho}$ is $\rho$-complete if and only if any $\rho$-Cauchy sequence is $\rho$-convergent

(4) A subset $C$ of $X_{\rho}$ is $\rho$-closed if the $\rho$-limit of a $\rho$-convergent sequence of $C$ always belong to $C$

(5) A subset $C$ of $X_{\rho}$ is $\rho$-bounded if we have $\delta_{\rho}(C)=\sup \{\rho(x-y): x, y \in C\}<\infty$

(6) A subset $K$ of $X_{\rho}$ is $\rho$-sequentially compact if any sequence $\left(x_{n}\right)_{n}$ of $C$ has a subsequence $\rho$-convergent to a point $x \in C$

(7) We say that $\rho$ satisfies the Fatou property if $\rho(x-y)$ $\leq \lim _{n \rightarrow+\infty} \rho\left(x-y_{n}\right)$ whenever $\left(y_{n}\right)_{n} \rho$-converges to $y$ for any $x, y$, and $y_{n}$ in $X_{\rho}$

Note that $\rho$-convergence does not imply $\rho$-Cauchy condition. Also, we have $x_{n} \longrightarrow^{\rho} x$ which does not imply in general $\lambda x_{n} \longrightarrow \rho \lambda x$ for every $\lambda>1$

An important property associated with a modular which plays a powerful role in modular spaces is the properties $\Delta_{2}$-condition and $\Delta_{2}$-type condition.

Definition 3 (see $[26,27])$. Let $\rho$ be a modular defined on a vector space $X$. We say that $\rho$ satisfies

(i) the $\Delta_{2}$-condition, if $\rho\left(2 x_{n}\right) \longrightarrow 0$ whenever $\rho\left(x_{n}\right)$ $\longrightarrow 0$ as $n$ goes to $+\infty$

(ii) the $\Delta_{2}$-type condition, if there exists $K>0$ such that $\rho(2 x) \leq K \rho(x)$ for all $x \in X_{\rho}$

If $\rho$ is assumed to be convex and to satisfy $\Delta_{2}$-type condition, we can define the growth function (see [28]):

$$
\begin{gathered}
\omega:[0, \infty) \longrightarrow[0, \infty), \\
\omega(t)=\sup \left\{\frac{\rho(t x)}{\rho(x)}: 0<\rho(x)<\infty\right\} .
\end{gathered}
$$

Then, $1<\omega(2)$. The arguments for this statement can be extracted from Lemma 2.6 in [10]. The minimal possible value of $K$ is usually denoted by $\omega(2)$.

In the sequel, we assume that $\rho$ is a convex modular.

Definition 4 (see [26]). Let $\rho$ be a modular and $r>0$ and $\varepsilon>0$. Define, for $i \in\{1,2\}$, that

$$
D_{i}(r, \varepsilon)=\left\{(x, y) \in X_{\rho} \times X_{\rho}: \rho(x) \leq r, \rho(y) \leq r, \rho\left(\frac{x-y}{i}\right) \geq r \varepsilon\right\} .
$$


If $D_{i}(r, \varepsilon) \neq \varnothing$, let

$$
\delta_{i}(r, \varepsilon)=\inf \left\{1-\frac{1}{r} \rho\left(\frac{x+y}{2}\right):(x, y) \in D_{i}\right\} .
$$

If $D_{i}(r, \varepsilon)=\varnothing$, we set $\delta_{i}(r, \varepsilon)=1$ :

(i) We say that $\rho$ is uniformly convex (UCi) if for every $r>0$ and $\varepsilon>0$, we have $\delta_{i}(r, \varepsilon)>0$

(ii) We say that $\rho$ is uniquely uniformly convex (UUCi) if for all $s \geq 0$ and $\varepsilon>0$, there exists $\eta(s, \varepsilon)>0$ such that $\delta_{i}(r, \varepsilon)>\eta(s, \varepsilon)$, for $r>s$

(iii) We say that $\rho$ is strictly convex (SC), if for every $x, y \in X_{\rho}$ such that $\rho(x)=\rho(y)$ and $\rho((x+y) / 2)=(\rho(x)+\rho(y)) / 2$, we have $x=y$

The following proposition characterizes the relationship between the above notions.

Proposition 5 (see [26]).

(a) (UUCi) implies (UCi) for $i=1,2$

(b) $\delta_{1}(r, \varepsilon) \leq \delta_{2}(r, \varepsilon)$ for $r>0$ and $\varepsilon>0$

(c) (UC1) implies (UC2) implies (SC)

(d) (UUC1) implies (UUC2)

The following property plays a similar role as the reflexivity in Banach spaces for modular spaces.

Definition 6 (see [27]). Let $\rho$ be a modular. We say that the modular space $X_{\rho}$ satisfies the property $(R)$ if and only if every decreasing sequence $\left(C_{n}\right)_{n \in \mathbb{N}}$ of nonempty $\rho$-closed convex and $\rho$-bounded subsets of $X_{\rho}$ has a nonempty intersection.

Lemma 7 (see [26]. Let $\rho$ be a convex modular that satisfies the Fatou property. Assume that $X_{\rho}$ is $\rho$-complete and $\rho$ is (UUC2). Then, $X_{\rho}$ satisfies the property $(R)$.

Definition 8 [26]. Let $\left(x_{n}\right)_{n}$ be a sequence in $X_{\rho}$ and $K$ be a nonempty subset of $X_{\rho}$. The function $\tau: K \longrightarrow[0, \infty]$, defined by $\tau(x)=\limsup _{n \rightarrow \infty} \rho\left(x_{n}-x\right)$, is called a $\rho$-type function.

Note that the $\rho$-type function $\tau$ is convex since $\rho$ is convex. A sequence $\left(c_{n}\right)_{n} \subset K$ is called a minimizing sequence of $\tau$ if $\lim _{n \rightarrow+\infty} \tau\left(c_{n}\right)=\inf _{x \in K} \tau(x)$.

The following lemmas play an important role in the proof of the next fixed point theorem.

Lemma 9 (see [26]). Let $\rho$ be a convex modular (UUC1) satisfying the Fatou property and $X_{\rho}$ a $\rho$-complete modular space. Let $C$ be a nonempty $\rho$-closed convex subset of $X_{\rho}$. Consider the $\rho$-type function $\tau: C \longrightarrow[0,+\infty]$ generated by a sequence $\left(x_{n}\right)_{n}$ in $X_{\rho}$. Assume that $\tau_{0}=\inf \{\tau(x): x \in C\}<\infty$. Then, all the minimizing sequences of $\tau$ are $\rho$-convergent to the same limit.

Lemma 10 (see [29]). Suppose that $\rho$ is convex and satisfies the property (UUC1), and let $\left(t_{n}\right)_{n}$ be a sequence in $[a, b]$, where $0<a \leq t_{n} \leq b<1$. If there exists a positive real number $r$ such that $\limsup _{n \rightarrow \infty} \rho\left(x_{n}\right) \leq r, \limsup _{n \rightarrow \infty} \rho\left(y_{n}\right) \leq r$, and $\lim _{n \rightarrow \infty} \rho\left(\left(1-t_{n}\right) x_{n}+t_{n} y_{n}\right)=r$, then $\lim _{n \rightarrow \infty} \rho\left(x_{n}-y_{n}\right)=0$.

Lemma 11 (see [30]). Let $\left(r_{n}\right)_{n}$ and $\left(t_{n}\right)_{n}$ be two real bounded sequences. Then,

(i) $\limsup _{n \rightarrow \infty} \max \left\{r_{n}, t_{n}\right\}=\max \left\{\limsup _{n \rightarrow \infty} r_{n}\right.$, $\left.\limsup _{n \rightarrow \infty} t_{n}\right\}$

(ii) let $z_{n}=\left(1-\alpha_{n}\right) r_{n}+\alpha_{n} t_{n}$, with $\left(\alpha_{n}\right)_{n} \subset(0,1)$ convergent to a real $\alpha \in(0,1)$. Then,

$$
\limsup _{n \rightarrow \infty} z_{n} \leq(1-\alpha) \limsup _{n \rightarrow \infty} r_{n}+\alpha \limsup _{n \rightarrow \infty} t_{n}
$$

Let us finish this section with a few of graph theory terminology and basic definitions which will be needed throughout. Let $C$ be a nonempty subset of a modular space $X_{\rho}$ and $\Delta=\{(x, x): x \in C\}$ be the loop set. Consider a directed graph $G$ such that the set of vertices $V(G)$ coincides with $C$ and the set of its edges $E(G)$ contains all loops, i.e., $\Delta \subset E(G)$. Assume that $G$ has no parallel edges, so it can be identified to the pair $(V(G), E(G))$.

Let $x$ and $y$ be vertices of a graph $G$. A path from $x$ to $y$ of length $N \in \mathbb{N}$ is a finite sequence $\left(x_{n}\right)$ of $N+1$ elements for which $x_{0}=x, x_{N}=y$, and $\left(x_{i}, x_{i+1}\right) \in E(G)$ for $i=0, \cdots, N-1$.

A graph $G$ is said to be connected if there is a path between any two vertices of the graph $G$. A directed graph $G=(V(G), E(G))$ is said to be transitive if for any $x, y$, and $z$ in $V(G)$ such that $(x, y) E(G)$ and $(y, z)$ are in $E(G)$, then $(x, z) \in E(G)$. Moreover, the conversion of a graph $G$, denoted $G^{-1}$, is the graph obtained by reversing the direction of the edges of the graph $G$. Thus, we have $E\left(G^{-1}\right)=\{(y, x)$ $\in X \times X:(x, y) \in E(G)\}$. For more details on graph theory, we refer readers to the book [31].

Definition 12. Let $X_{\rho}$ be a modular space. A graph $G$ is said to be convex if and only if for any $x, y, z$, and $w$ in $X_{\rho}$ and $\lambda \in[0,1]$, we have $(x, y) \in E(G)$ and $(z, w) \in E(G)$ which lead to $((1-\lambda) x+\lambda z,(1-\lambda) y+\lambda w) \in E(G)$.

Definition 13. Let $C$ be a nonempty subset of a modular space $X_{\rho}$, and let $G=(V(G), E(G))$ be a directed graph such that $V(G)=C$. We say that $C$ have the property $(P)$ : if each sequence $\left(x_{n}\right)_{n}$ in $C \rho$-converges to $x$ and $\left(x_{n}, x_{n+1}\right) \in E(G)$, then $\left(x_{n}, x\right) \in E(G)$ for all $n \geq 0$. 


\section{Results and Discussion}

Throughout, we assume that $X_{\rho}$ is a modular space, where $\rho$ is a convex modular. Let $C$ be a nonempty $\rho$-closed convex subset of $X_{\rho}$. Let $G=(V(G), E(G))$ be a directed transitive graph such that $V(G)=C, E(G)$ is convex and $\Delta \subseteq E(G)$. Moreover, we assume that $G$-intervals are $\rho$-closed. Recall that a $G$-interval is any of the subsets $[a, \longrightarrow)=$ $\{x \in X:(a, x) \in E(G)\}, \quad(\longleftarrow, b]=\{x \in X:(x, b) \in E(G)\}$, and $[a, b]=[a, \longrightarrow) \cap(\longleftarrow, b]$.

Definition 14. Let $C$ be a nonempty subset of a modular space $X_{\rho}$. Let $T: C \longrightarrow C$ be a mapping and $\gamma \in[0,1]$ and $\mu \in[0$, $1 / 2$ ] such that $2 \mu \leq \gamma$. The mapping $T$ is said to

(1) preserve edges if $(T x, T y) \in E(G)$ whenever $(x, y) \in E(G)$ for any $x, y \in C$

(2) satisfy the condition $G-\mathscr{M}_{\gamma, \mu}$ if $T$ preserves edges $\gamma \rho(x-T x) \leq \rho(x-y)+\mu \rho(y-T y)$ which leads to $\rho(T x-T y) \leq(1-\gamma) \rho(x-y)+\rho(\mu(x-T y))+\rho(\mu(y$ $-T x)$ ), for all $x, y \in C$ such that $(x, y) \in E(G)$

Note that if $\gamma=\mu=0$, then $T$ is $\rho$-nonexpansive.

Lemma 15. Let $\rho$ be a convex modular and $X_{\rho}$ be a modular space endowed with a directed transitive graph such that $V(G)=C, E(G)$ is convex and $\Delta \subseteq E(G)$. Let $T: C \longrightarrow C$ satisfy the condition $G-M_{\gamma, \mu}$ on $C$. If $z$ is a fixed point of $T$, then $\rho(z-T x) \leq \rho(z-x)$ for all $x \in C$ such that $(x$, $z) \in E(G)$

Proof. Let $z$ be a fixed point of $T$ and $x \in C$ such that $(x, z)$ $\in E(G)$. We have

$$
0=\rho(z-T z) \leq \rho(x-z) \leq \rho(x-z)+\mu \rho(x-T x) .
$$

By condition $G-\mathscr{M}_{\gamma, \mu}$,

$$
\begin{aligned}
\rho(T x-T z)= & \rho(z-T x) \leq(1-\gamma) \rho(x-z)+\rho(\mu(x-T z)) \\
& +\rho(\mu(z-T x)) \leq(1-\gamma) \rho(x-z) \\
& +\mu \rho(x-z)+\mu \rho(z-T x) .
\end{aligned}
$$

Hence, $\quad(1-\mu) \rho(z-T x) \leq(1-\gamma+\mu) \rho(z-x), \quad$ which implies

$$
\rho(z-T x) \leq \frac{1-\gamma+\mu}{1-\mu} \rho(z-x)
$$

Since $2 \mu \leq \gamma$, then $\rho(z-T x) \leq \rho(z-x)$.

It is easy to obtain the following consequence of condition $\left(3^{\prime}\right)$, which we need in the sequel, by using the convexity of the modular $\rho$, and we prove by induction on $n \geq 1$ the inequality of the property.
Property 16. Let $\rho$ be a convex modular. For $a_{1}, \cdots, a_{n}$ in $\mathbb{R}_{+}$ such that $\sum_{i=1}^{n} a_{i}=1$ and $x_{1}, \cdots, x_{n}$ in $X_{\rho}$, we have

$$
\rho\left(\sum_{i=1}^{n} a_{i} x_{i}\right) \leq \sum_{i=1}^{n} a_{i} \rho\left(x_{i}\right) .
$$

Proposition 17. Let $\rho$ be a convex modular and $X_{\rho}$ be a modular space endowed with a directed transitive graph such that $V(G)=C, E(G)$ is convex and $\Delta \subseteq E(G)$. Let $T$ be a selfmapping on $C$ monotone and satisfy the condition $G-\mathscr{M}_{\gamma, \mu}$ on $C$, where $\gamma \leq 1 /(\omega(2))$. Then,

(i) for all $x \in C$ such that $(x, T x) \in E(G)$, we have $\rho\left(T x-T^{2} x\right) \leq \rho(x-T x)$

(ii) if $\rho$ satisfies the $\Delta_{2}$-type condition, then for all $x$ and $y$ in $C$ such that $(x, T x)$ and $(T x, y)$ are in $E(G)$ and for $c \in[0,1]$, we have $(a)$ or $(b)$ :
(a) $(c /(\omega(2))) \rho(x-T x) \leq \rho(x-y)$
(b) $(c /(\omega(2))) \rho\left(T x-T^{2} x\right) \leq \rho(T x-y)$

Proof.

(i) Let $x \in C$ such that $(x, T x) \in E(G)$. Then, we have $\gamma \rho$ $(x-T x) \leq \rho(x-T x) \leq \rho(x-T x)+\mu \rho\left(T x-T^{2} x\right)$. By the condition $G-\mathscr{M}_{\gamma, \mu}$ for $y=T x$, we have

$$
\begin{aligned}
\rho\left(T x-T^{2} x\right) \leq & (1-\gamma) \rho(x-T x)+\rho\left(\mu\left(x-T^{2} x\right)\right) \\
& +\rho(\mu(T x-T x)) \leq(1-\gamma) \rho(x-T x) \\
& +\rho\left(\mu\left(x-T x+T x-T^{2} x\right)\right) \\
\leq & (1-\gamma) \rho(x-T x)+\mu \rho(x-T x) \\
& +\mu \rho\left(T x-T^{2} x\right)
\end{aligned}
$$

which implies $(1-\mu) \rho\left(T x-T^{2} x\right) \leq(1-\gamma+\mu) \rho(x-T x)$. Since $2 \mu \leq \gamma$, then $\rho\left(T x-T^{2} x\right) \leq \rho(x-T x)$.

(ii) Assume that $\rho$ satisfies the $\Delta_{2}$-type condition, that is, $\rho(2 x) \leq \omega(2) \rho(x)$ for all $x \in X_{\rho}$. Let $x, y \in C$ such that $(x, T x)$ and $(T x, y)$ are in $E(G)$. Assume on the contrary that $(c /(\omega(2))) \rho(x-T x)>\rho(x-y)$ and $(c /(\omega(2))) \rho\left(T x-T^{2} x\right)>\rho(T x-y)$. We have

$$
\begin{aligned}
\rho(x-T x) & \leq \frac{\omega(2)}{2} \rho(x-y)+\frac{\omega(2)}{2} \rho(T x-y) \\
& <\frac{c}{\omega(2)} \frac{\omega(2)}{2} \rho\left(T x-T^{2} x\right)+\frac{c}{\omega(2)} \frac{\omega(2)}{2} \rho(x-T x) \\
& \leq c \rho(x-T x) \leq \rho(x-T x) .
\end{aligned}
$$

Then, $\rho(x-T x)<\rho(x-T x)$ which is impossible. Therefore, (a) or (b) holds. 
Let $T: C \longrightarrow C$ be a mapping preserving edges. Let $\left(\alpha_{n}\right)_{n}$ be a sequence $(0,1)$. Let $x_{0} \in C$ such that $\left(x_{0}, T x_{0}\right) \in E(G)$ (resp., $\left(T x_{0}, x_{0}\right) \in E(G)$ ). By induction, we construct a sequence $\left(x_{n}\right)_{n}$ in $C$, called the Mann iteration, defined by

$$
x_{n+1}=\left(1-\alpha_{n}\right) x_{n}+\alpha_{n} T x_{n} .
$$

Recall that $\left(x_{n}, x_{n+1}\right),\left(x_{n}, T x_{n}\right)$, and $\left(T x_{n}, T x_{n+1}\right)$ (resp., $\left(x_{n+1}, x_{n}\right),\left(T x_{n}, x_{n}\right)$, and $\left.\left(T x_{n+1}, T x_{n}\right)\right)$ are in $E(G)$, for any $n \geq 0$.

In the following result, we prove the existence of fixed points of $G-\mathscr{M}_{\gamma, \mu}$ mappings using the Mann iteration defined by (14) such that the sequence $\left(\alpha_{n}\right)_{n}$ converges to $\alpha \in(0,1)$.

Theorem 18. Let $\rho$ be a (UUC1) convex modular that satisfies the Fatou property and the $\Delta_{2}$-type condition, and $X_{\rho}$ is a $\rho$-complete modular space endowed with a directed transitive graph, where $V(G)=C$ is a nonempty $\rho$-closed convex and $\rho$-bounded, $E(G)$ is convex, and $\Delta \subseteq E(G)$. Let $T: C$

$\longrightarrow C$ be a mapping satisfying the condition $G-\mathscr{M}_{\gamma, \mu}$ with $\gamma \in[0,1 /(\omega(2))]$ and $\rho$-continuous on $C$. Assume that there exists $x_{0} \in C$ such that $\left(x_{0}, T x_{0}\right) \in E(G)$ (resp., (Tx $x_{0}$, $\left.x_{0}\right) \in E(G)$ ). Then, $T$ has a fixed point $z$ in $C$ such that $\left(x_{0}, z\right) \in E(G)$ (resp., $\left.\left(z, x_{0}\right) \in E(G)\right)$ if and only if $\lim _{n \rightarrow \infty}$ $\rho\left(x_{n}-T x_{n}\right)=0$.

Proof. Assume that $T$ has a fixed point $p \in C$ such that $\left(x_{0}, p\right) \in E(G)$. Using the convexity of the modular $\rho$, we have

$$
\begin{aligned}
\rho\left(x_{n+1}-p\right) & \leq\left(1-\alpha_{n}\right) \rho\left(x_{n}-p\right)+\alpha_{n} \rho\left(T x_{n}-p\right) \\
& \leq\left(1-\alpha_{n}\right) \rho\left(x_{n}-p\right)+\alpha_{n} \rho\left(x_{n}-p\right) \leq \rho\left(x_{n}-p\right) .
\end{aligned}
$$

It follows that $\left(\rho\left(x_{n}-p\right)\right)_{n}$ is a decreasing nonnegative sequence. Since $C$ is convex, then $x_{n} \in C$ for all $n \in$ $\mathbb{N}$. Moreover, $C \rho$-bounded implies that $\rho\left(x_{n}-p\right)<\infty$ for all $n \in \mathbb{N}$. These lead to the conclusion that $\left(\rho\left(x_{n}-p\right)\right)_{n}$ is convergent. Let $\lim _{n} \rho\left(x_{n}-p\right)=r$. We have $\rho\left(T x_{n}-p\right) \leq \rho\left(x_{n}-p\right)$. Then,

$$
\limsup _{n \rightarrow \infty} \rho\left(T x_{n}-p\right) \leq \limsup _{n \rightarrow \infty} \rho\left(x_{n}-p\right)=r .
$$

Moreover,

$\lim _{n \rightarrow \infty} \rho\left(\left(1-\alpha_{n}\right)\left(x_{n}-p\right)+\alpha_{n}\left(T x_{n}-p\right)\right)=\lim _{n \rightarrow \infty} \rho\left(x_{n+1}-p\right)=r$.

Thus, by Lemma 10, we get $\lim _{n \rightarrow \infty} \rho\left(x_{n}-T x_{n}\right)=0$. In the following, let us prove the converse statement. Let $x_{0} \in C$ such that $\left(x_{0}, T x_{0}\right) \in E(G)$. Set $K=\left\{y \in C:\left(x_{n}\right.\right.$, $y) \in E(G), \forall n \geq 0\}$. The subset $K$ is a nonempty $\rho$-closed convex. In fact, set $C_{n}=\left\{y \in C:\left(x_{n}, y\right) \in E(G)\right\}$ for all $n$ $\geq 0$. We have $\left(C_{n}\right)_{n}$ which is a decreasing sequence of non- empty $\rho$-closed convex $\rho$-bounded subsets of $X_{\rho}$. Indeed, it is easy to verify that $C_{n}$ is nonempty and $\rho$-bounded for all $n \geq 0$. Let $\lambda \in(0,1)$ and $y_{1}, y_{2} \in C_{n}$. Then, $\left(x_{n}, \lambda y_{1}+\right.$ $\left.(1-\lambda) y_{2}\right) \in E(G)$. Hence, $\lambda y_{1}+(1-\lambda) y_{2} \in C_{n}$; that is, $C_{n}$ is convex for all $n \geq 0$. Moreover, $C_{n}$ is $\rho$-closed for all $n \geq 0$. In fact, let $\left(y_{p}\right)_{p} \subset C_{n} \rho$-converge to $y \in X_{\rho}$. By the definition of $C_{n}$, we have $C_{n}=C \cap\left[x_{n}, \longrightarrow\right)$. Since $C$ and $\left[x_{n}, \longrightarrow\right)$ are supposed to be $\rho$-closed, then $y \in C_{n}$ for all $n \in \mathbb{N}$. Since $\left(x_{n}, x_{n+1}\right) \in E(G)$, then $C_{n+1} \subset C_{n}$; that is, $\left(C_{n}\right)_{n}$ is a decreasing sequence. By Lemma 7 , the modular space $X_{\rho}$ satisfies the property $(R)$. Then, $K=\bigcap_{n} C_{n}$ is a nonempty $\rho$-closed convex.

Let $\tau, \bar{\tau}: K \longrightarrow[0,+\infty]$ be two $\rho$-type functions generated by the sequences $\left(x_{n}\right)_{n}$ and $\left(T x_{n}\right)_{n}$, respectively; that is, $\tau(y)=\limsup _{n \rightarrow \infty} \rho\left(x_{n}-y\right)$ and $\bar{\tau}(y)=\limsup _{n \rightarrow \infty} \rho\left(T x_{n}\right.$ $-y)$. Let us prove that for all $y \in K$ such that $\left(x_{n}, y\right) \in E(G)$ and $\left(T x_{n}, y\right) \in E(G)$, one has

$$
\tau(T y) \leq \bar{\tau}(T y) \leq \tau(y) \leq \bar{\tau}(y) .
$$

Case 1. If $\left(x_{n}\right)_{n}$ satisfies (ii, a) of Proposition 17 for some $n$ $\geq 0$, that is, $(c /(\omega(2))) \rho\left(x_{n}-T x_{n}\right) \leq \rho\left(x_{n}-y\right)$, then by condition $G-\mathscr{M}_{\gamma, \mu}$ for $c=\gamma \omega(2) \in[0,1]$, we have

$$
\begin{aligned}
\rho\left(T x_{n}-T y\right) \leq & (1-\gamma) \rho\left(x_{n}-y\right)+\rho\left(\mu\left(T x_{n}-y\right)\right) \\
& +\rho\left(\mu\left(T y-x_{n}\right)\right) \leq(1-\gamma) \rho\left(x_{n}-y\right)+\mu \rho\left(x_{n}-y\right) \\
& +\mu \rho\left(T x_{n}-x_{n}\right)+\mu \rho\left(x_{n}-T x_{n}\right)+\mu \rho\left(T x_{n}-T y\right),
\end{aligned}
$$

for $n \geq 0$. Then,

$$
(1-\mu) \rho\left(T x_{n}-T y\right) \leq(1-\gamma+\mu) \rho\left(x_{n}-y\right)+2 \mu \rho\left(x_{n}-T x_{n}\right) \text {, }
$$

for all $n \geq 0$. Hence,

$$
\begin{aligned}
\rho\left(T x_{n}-T y\right) & \leq \frac{(1-\gamma+\mu)}{(1-\mu)} \rho\left(x_{n}-y\right)+\frac{2 \mu}{(1-\mu)} \rho\left(x_{n}-T x_{n}\right) \\
& \leq \rho\left(x_{n}-y\right)+\frac{2 \mu}{(1-\mu)} \rho\left(x_{n}-T x_{n}\right),
\end{aligned}
$$

for all $n \geq 0$, since $2 \mu \leq \gamma$.

Case 2. If $\left(x_{n}\right)_{n}$ satisfies (ii, b) of Proposition 17 for some $n$ $\geq 0$, that is,

$$
\begin{aligned}
\frac{c}{\omega(2)} \rho\left(T y_{n}-y_{n}\right) & =\frac{c}{\omega(2)} \rho\left(T^{2} x_{n}-T x_{n}\right) \\
& \leq \rho\left(T x_{n}-y\right)=\rho\left(y_{n}-y\right), \\
\frac{c}{\omega(2)} \rho\left(T x_{n}-x_{n}\right) & >\rho\left(x_{n}-y\right),
\end{aligned}
$$

where $y_{n}=T x_{n}$. By the condition $G-\mathscr{M}_{\gamma, \mu}$ for $c=\gamma \omega(2)$ $\in[0,1]$, we have 


$$
\begin{aligned}
\rho\left(T y_{n}-T y\right)= & \rho\left(T^{2} x_{n}-T y\right) \leq(1-\gamma) \rho\left(y_{n}-y\right) \\
& +\rho\left(\mu\left(T y_{n}-y\right)\right)+\rho\left(\mu\left(y_{n}-T y\right)\right) \\
\leq & (1-\gamma) \rho\left(y_{n}-y\right)+\mu \rho\left(y_{n}-T y_{n}\right) \\
& +\mu \rho\left(T y_{n}-T y\right)+\mu \rho\left(y-y_{n}\right)+\mu \rho\left(y_{n}-T y_{n}\right),
\end{aligned}
$$

for all $n \geq 0$. It follows that $(1-\mu) \rho\left(T y_{n}-T y\right) \leq(1-\gamma+$ $\mu) \rho\left(y_{n}-y\right)+2 \mu \rho\left(y_{n}-T y_{n}\right)$ for all $n \geq 0$. Since $2 \mu \leq \gamma$, then

$$
\rho\left(T y_{n}-T y\right) \leq \rho\left(y_{n}-y\right)+\frac{2 \mu}{1-\mu} \rho\left(y_{n}-T y_{n}\right)
$$

for all $n \geq 0$. Moreover, for all $n \geq 0$,

$$
\begin{aligned}
\rho\left(T x_{n}-T y\right)= & \rho\left(y_{n}-T y\right) \leq \frac{\omega(2)}{2}\left[\rho\left(y_{n}-T y_{n}\right)+\rho\left(T y_{n}-T y\right)\right] \\
\leq & \frac{\omega(2)}{2}\left[\rho\left(y_{n}-T y_{n}\right)+\rho\left(y_{n}-y\right)+\frac{2 \mu}{1-\mu} \rho\left(y_{n}-T y_{n}\right)\right] \\
\leq & \frac{\omega(2)}{2}\left[\rho\left(y_{n}-y\right)+\frac{1+\mu}{1-\mu} \rho\left(y_{n}-T y_{n}\right)\right] \\
\leq & \frac{\omega(2)}{2}\left[\frac{1+\mu}{1-\mu} \rho\left(y_{n}-T y_{n}\right)+\frac{\omega(2)}{2} \rho\left(y_{n}-x_{n}\right)\right. \\
& \left.+\frac{\omega(2)}{2} \rho\left(x_{n}-y\right)\right]<\frac{\omega(2)}{2}\left[\frac{1+\mu}{1-\mu} \rho\left(y_{n}-T y_{n}\right)\right. \\
& \left.+\frac{\omega(2)}{2} \rho\left(y_{n}-x_{n}\right)+\frac{\omega(2)}{2} \frac{c}{\omega(2)} \rho\left(y_{n}-x_{n}\right)\right] \\
\leq & \frac{\omega(2)}{2}\left[\frac{1+\mu}{1-\mu} \rho\left(y_{n}-T y_{n}\right)+\frac{c+\omega(2)}{2} \rho\left(x_{n}-y_{n}\right)\right] .
\end{aligned}
$$

By Proposition 17, we have $\rho\left(T y_{n}-y_{n}\right)=\rho\left(T^{2} x_{n}-T\right.$ $\left.x_{n}\right) \leq \rho\left(x_{n}-T x_{n}\right)$. Then,

$$
\begin{aligned}
\rho\left(y_{n}-T y\right)= & \rho\left(T x_{n}-T y\right)<\frac{\omega(2)}{2}\left[\frac{1+\mu}{1-\mu} \rho\left(x_{n}-T x_{n}\right)\right. \\
& \left.+\frac{c+\omega(2)}{2} \rho\left(x_{n}-T x_{n}\right)\right] \\
< & \frac{\omega(2)}{2}\left(3+\frac{\omega(2)+c}{2}\right) \rho\left(x_{n}-T x_{n}\right),
\end{aligned}
$$

for all $n \geq 0$. Thus,

$\rho\left(y_{n}-T y\right)=\rho\left(T x_{n}-T y\right)<\frac{\omega(2)(6+c+\omega(2))}{4} \rho\left(x_{n}-T x_{n}\right)$,

for all $n \geq 0$. Therefore, by (21) and (27), we get

$$
\begin{gathered}
\rho\left(y_{n}-T y\right)=\rho\left(T x_{n}-T y\right)<\max \left\{\rho\left(x_{n}-y\right)+\frac{2 \mu}{(1-\mu)}\right. \\
\left.\cdot \rho\left(x_{n}-T x_{n}\right), \frac{\omega(2)(6+c+\omega(2))}{4} \rho\left(x_{n}-T x_{n}\right)\right\} .
\end{gathered}
$$

By the limsup as $n$ goes to infinity, we have

$$
\bar{\tau}(T y) \leq \max \{\tau(y), 0\}=\tau(y),
$$

since $\lim _{n \rightarrow \infty} \rho\left(x_{n}-T x_{n}\right)=0$. Otherwise, $\rho\left(x_{n+1}-y\right) \leq \alpha_{n} \rho$ $\left(T x_{n}-y\right)+\left(1-\alpha_{n}\right) \rho\left(x_{n}-p\right)$ for all $n \geq 0$. By limsup as $n$ goes to infinity, we get

$$
\tau(y) \leq \bar{\tau}(y)
$$

Thus, by (29) and (30), one has

$$
\tau(T y) \leq \bar{\tau}(T y) \leq \tau(y) \leq \bar{\tau}(y)
$$

for all $y \in K$. Let $\left(z_{p}\right)_{p}$ be a minimizing sequence of the type function $\tau$. By Lemma $9,\left(z_{p}\right)_{p} \rho$-converges to a point $z \in K$. By (31), the sequence $\left(T z_{p}\right)_{p}$ is also a minimizing sequence of $\tau$. Hence, $\left(T z_{p}\right)_{p}$ converges also to $z$. Since $T$ is $\rho$-continuous, then $\left(T z_{p}\right)_{p} \rho$-converges to $T z$. Thus, the uniqueness of the limit implies that $z=T z$.

Example 1. For the function $p: \mathbb{N} \longrightarrow[1, \infty)$, consider the modular space

$\ell_{p(\cdot)}=\left\{\left(x_{n}\right)_{n} \in \mathbb{R}^{\mathbb{N}}: \sum_{n=0}^{+\infty} \frac{1}{p(n)}\left|\lambda x_{n}\right|^{p(n)}<\infty \quad\right.$ for some $\left.\lambda>0\right\}$,

where the modular $\rho$ is given by $\rho(x)=\rho\left(\left(x_{n}\right)_{n}\right)=\sum_{n=0}^{+\infty}(1 /$ $(p(n)))\left|x_{n}\right|^{p(n)}$ such that $1<p^{-} \leq p(n) \leq p^{+}<\infty$ for all $n \geq 0$, where $p^{-}=\inf _{n \in \mathbb{N}} p(n)$ and $p^{+}=\sup _{n \in \mathbb{N}} p(n)$.

We have $\rho$ which is a convex modular satisfying the Fatou property and (UUC2) (for more details, see [32]). Moreover, $\rho$ satisfies the $\Delta_{2}$-type condition. In fact, let $x \in \ell_{p(.)}$. Since $p(n) \leq p^{+}$, we have $2^{p(n)} \leq 2^{p^{+}}$. Thus, $\left(2^{p^{(n)}} /\right.$ $(p(n)))\left|x_{n}\right|^{p(n)} \leq\left(2^{p^{+}} /(p(n))\right)\left|x_{n}\right|^{p(n)}$. Therefore, $\sum_{n=0}^{q}\left(2^{p(n)} /\right.$ $(p(n)))\left|x_{n}\right|^{p(n)} \leq \sum_{n=0}^{q}\left(2^{p^{+}} /(p(n))\right)\left|x_{n}\right|^{p(n)}$ for all $q \in \mathbb{N}$. Taking $\lim _{n \rightarrow \infty}$, one has

$$
\begin{aligned}
\rho(2 x) & =\sum_{n=0}^{+\infty} \frac{2^{p(n)}}{p(n)}\left|x_{n}\right|^{p(n)} \leq \sum_{n=0}^{+\infty} \frac{2^{p^{+}}}{p(n)}\left|x_{n}\right|^{p(n)} \\
& \leq 2^{p^{+}} \sum_{n=0}^{+\infty} \frac{1}{p(n)}\left|x_{n}\right|^{p(n)}=2^{p^{+}} \rho(x) .
\end{aligned}
$$

Recall that if $\rho$ satisfies the $\Delta_{2}$-type condition, then $\|\cdot\|_{\rho}$ convergence is equivalent to $\rho$-convergence (see [33]). Since 
$\left(\ell_{p(.)},\|\cdot\|_{\rho}\right)$ is a Banach space (see [32]), then $\ell_{p(.)}$ is a $\rho$-complete modular space.

Recall the following two inequalities:

(i) For all $1<p \leq 2$, we have

$$
\left|\frac{a+b}{2}\right|^{p}+\frac{p(p-1)}{2}\left|\frac{a-b}{|a|+|b|}\right|^{2-p}\left|\frac{a-b}{2}\right|^{p} \leq \frac{1}{2}\left(|a|^{p}+|b|^{p}\right),
$$

for all $a, b \in \mathbb{R}$ such that $|a|+|b| \neq 0$.

(ii) For all $p \geq 2$, we have

$$
\left|\frac{a+b}{2}\right|^{p}+\left|\frac{a-b}{2}\right|^{p} \leq \frac{1}{2}\left(|a|^{p}+|b|^{p}\right),
$$

for all $a, b \in \mathbb{R}$.

The modular $\rho$ is (UUC1). In fact, let $r>0, \varepsilon>0$, and $x$, $y \in \ell_{p(\cdot)}$ such that $\rho(x) \leq r, \rho(y) \leq r$, and $\rho(x-y) \geq r \varepsilon$. Let us partition the set $\mathbb{N}$ into two subsets $E$ and $F$ given as follows: $E=\{n \in \mathbb{N}: p(n) \geq 2\}$ and $F=\{n \in \mathbb{N}: p(n)<2\}$. Using the definition of the modular $\rho$, we will denote $\rho(x)=\rho_{E}(x)$ $+\rho_{F}(x)$, because the two sums on $E$ and $F$ converge, with

$$
\begin{aligned}
& \rho_{E}(x)=\sum_{n \in E} \frac{1}{p(n)}\left|x_{n}\right|^{p(n)} \\
& \rho_{F}(x)=\sum_{n \in F} \frac{1}{p(n)}\left|x_{n}\right|^{p(n)},
\end{aligned}
$$

for all $x \in \ell_{p(\cdot)}$. Thus, $\rho(x-y) \geq r \varepsilon$ leads to $\rho_{E}(x-y) \geq r \varepsilon / 2$ or $\rho_{F}(x-y) \geq r \varepsilon / 2$.

Case 1. Assume that $\rho_{E}(x-y) \geq r \varepsilon / 2$. Since $p(n) \leq p^{+}<\infty$, then

$$
\rho_{E}\left(\frac{x-y}{2}\right) \geq \frac{1}{2^{p^{+}}} \rho_{E}(x-y) \geq \frac{1}{2^{p^{+}}} \frac{r \varepsilon}{2}=\frac{r \varepsilon}{2.2^{p^{+}}} .
$$

Since $p(n) \geq 2$, then by the inequality (ii), we get

$$
\rho_{E}\left(\frac{x+y}{2}\right)+\rho_{E}\left(\frac{x-y}{2}\right) \leq \frac{1}{2}\left(\rho_{E}(x)+\rho_{E}(y)\right) .
$$

By the convexity of $\rho_{F}$, we have

$$
\rho\left(\frac{x+y}{2}\right)+\rho_{E}\left(\frac{x-y}{2}\right) \leq \frac{1}{2}(\rho(x)+\rho(y)) \leq r .
$$

Thus,

$$
\rho\left(\frac{x+y}{2}\right) \leq r-\rho_{E}\left(\frac{x-y}{2}\right) \leq r-\frac{r \varepsilon}{2.2^{p^{+}}}=r\left(1-\frac{\varepsilon}{2.2^{p^{+}}}\right) .
$$

Case 2. Assume that $\rho_{F}(x-y) \geq r \varepsilon / 2$. Let $A$ be a subset of $F$ defined by $A=\left\{n \in F:\left|x_{n}-y_{n}\right|>c\left(\left|x_{n}\right|+\left|y_{n}\right|\right)\right\}$, where $\quad c=\min \{1 / 2 ; \varepsilon / 8\}$. Let $B=F \backslash A$. Then, $\left|x_{n}-y_{n}\right| \leq c\left(\left|x_{n}\right|+\left|y_{n}\right|\right)$ for all $n \in B$. Thus,

$$
\begin{aligned}
\sum_{n \in B} \frac{1}{p(n)}\left|x_{n}-y_{n}\right|^{p(n)} & \leq \sum_{n \in B} \frac{1}{p(n)}\left|c\left(\left|x_{n}\right|+\left|y_{n}\right|\right)\right|^{p(n)} \\
& \leq \sum_{n \in B} \frac{1}{p(n)}|2 c|^{p(n)}\left|\frac{\left|x_{n}\right|+\left|y_{n}\right|}{2}\right|^{p(n)} \\
& \leq \sum_{n \in B} \frac{1}{p(n)}|2 c|^{p(n)} \frac{\left|x_{n}\right|^{p(n)}+\left|y_{n}\right|^{p(n)}}{2} \\
& \leq \frac{1}{2}(\rho(2 c x)+\rho(2 c y)) \\
& \leq \frac{2 c}{2}(\rho(x)+\rho(y)) \quad \text { since } c \leq \frac{1}{2} \leq \frac{4 c r}{2}=2 c r .
\end{aligned}
$$

Hence,

Thus, $\sum_{n \in B}(1 /(p(n)))\left|x_{n}-y_{n}\right|^{p(n)} \leq 2 r c \leq r \varepsilon / 4$ since $c \leq \varepsilon / 8$.

$$
\begin{aligned}
& \sum_{n \in A} \frac{1}{p(n)}\left|x_{n}-y_{n}\right|^{p(n)}=\rho_{F}(x-y)-\sum_{n \in B} \frac{1}{p(n)}\left|x_{n}-y_{n}\right|^{p(n)} \\
& \quad \geq \frac{r \varepsilon}{2}-\frac{r \varepsilon}{4}=\frac{r \varepsilon}{4},
\end{aligned}
$$

since $B=F \backslash A$. Otherwise,

$$
\begin{gathered}
p(n)(p(n)-1) \geq p^{-}\left(p^{-}-1\right) \geq p^{-}-1, \\
\left|\frac{x_{n}-y_{n}}{\left|x_{n}\right|+\left|y_{n}\right|}\right|^{2-p(n)} \geq c^{2-p(n)} \geq c,
\end{gathered}
$$

for all $n \in A$. Thus, by the inequality (i),

$$
\left|\frac{x_{n}+y_{n}}{2}\right|^{p(n)}+\frac{c\left(p^{-}-1\right)}{2}\left|\frac{x_{n}-y_{n}}{2}\right|^{p(n)} \leq \frac{1}{2}\left(\left|x_{n}\right|^{p(n)}+\left|y_{n}\right|^{p(n)}\right),
$$

for all $n \in A$. Thus,

$$
\begin{aligned}
\sum_{n \in A} & \frac{1}{p(n)}\left|\frac{x_{n}+y_{n}}{2}\right|^{p(n)}+\frac{c\left(p^{-}-1\right)}{2} \sum_{n \in A} \frac{1}{p(n)}\left|\frac{x_{n}-y_{n}}{2}\right|^{p(n)} \\
& \leq \frac{1}{2}\left(\sum_{n \in A} \frac{1}{p(n)}\left|x_{n}\right|^{p(n)}+\sum_{n \in A} \frac{1}{p(n)}\left|y_{n}\right|^{p(n)}\right) .
\end{aligned}
$$

Hence,

$$
\rho_{A}\left(\frac{x+y}{2}\right)+\frac{c\left(p^{-}-1\right)}{2} \rho_{A}\left(\frac{x-y}{2}\right) \leq \frac{1}{2}\left(\rho_{A}(x)+\rho_{A}(y)\right) .
$$


Thus,

$\rho_{A}\left(\frac{x+y}{2}\right) \leq \frac{1}{2}\left(\rho_{A}(x)+\rho_{A}(y)\right)-\frac{c\left(p^{-}-1\right)}{2} \rho_{A}\left(\frac{x-y}{2}\right)$.

Since $p(n)<2$, then

$$
\begin{aligned}
\rho_{A}\left(\frac{x-y}{2}\right) & =\sum_{n \in A} \frac{1}{p(n)}\left|\frac{x_{n}-y_{n}}{2}\right|^{p(n)} \\
& \geq \frac{1}{4} \sum_{n \in A} \frac{1}{p(n)}\left|x_{n}-y_{n}\right|^{p(n)} \geq \frac{1}{4} \rho_{A}(x-y) .
\end{aligned}
$$

Therefore, $\rho_{A}((x+y) / 2) \leq(1 / 2)\left(\rho_{A}(x)+\rho_{A}(y)\right)-\left(\left(c\left(p^{-}\right.\right.\right.$ $-1)) / 2) \cdot\left(\left(\rho_{A}(x-y)\right) / 4\right)$. It follows

$$
\rho_{A}\left(\frac{x+y}{2}\right) \leq \frac{1}{2}\left(\rho_{A}(x)+\rho_{A}(y)\right)-\frac{\operatorname{crc}\left(p^{-}-1\right)}{32},
$$

since $\rho_{A}(x-y) \geq r \varepsilon / 4$. Let $\bar{A}=\mathbb{N} \backslash A$. Since $\rho_{\bar{A}}$ is convex, we have

$$
\begin{aligned}
\rho\left(\frac{x+y}{2}\right)= & \rho_{A}\left(\frac{x+y}{2}\right)+\rho_{\bar{A}}\left(\frac{x+y}{2}\right) \leq \frac{1}{2}\left(\rho_{A}(x)+\rho_{A}(y)\right) \\
& +\left(\rho_{\bar{A}}(x)+\rho_{\bar{A}}(y)\right)-\frac{\operatorname{crc}\left(p^{-}-1\right)}{32} \leq \frac{1}{2}(\rho(x) \\
& +\rho(y))-\frac{\operatorname{crc}\left(p^{-}-1\right)}{32} \leq r\left(1-\frac{\operatorname{crc}\left(p^{-}-1\right)}{32}\right) .
\end{aligned}
$$

Therefore, by (40) and (50), we put $\eta_{1}(s, \varepsilon)=\min \{\varepsilon / 2$. $\left.2^{p^{+}} ;\left(\varepsilon c\left(p^{-}-1\right)\right) / 32\right\}$. Thus, $\rho$ is (UUC1).

Consider the nonempty set $C=\left\{x=\left(x_{n}\right)_{n} \in \ell_{p(.)}: 0 \leq x_{n}\right.$ $\left.\leq 2^{-n}, \forall n \geq 0\right\}$. It is clear that $C$ is convex and $\rho$-bounded, and it is $\rho$-closed. Indeed, let $\left(x^{q}\right)_{q}$ be a sequence in $C$ which $\rho$-converges to a point $x=\left(x_{0}, x_{1}, \cdots\right) \in \ell_{p(\cdot)}$, where $x^{q}=\left(x_{0}^{q}\right.$, $\left.x_{1}^{q}, \cdots\right)$, for all $q \geq 0$. We have

$$
\frac{1}{p^{+}}\left|x_{n}^{q}-x_{n}\right|^{m} \leq \sum_{n=0}^{+\infty} \frac{1}{p(n)}\left|x_{n}^{q}-x_{n}\right|^{p(n)}
$$

for all $q \geq 0$, where $\left\{\begin{array}{l}m=p^{+} \text {if }\left|x_{n}^{q}-x_{n}\right| \geq 1 \\ m=p^{-} \text {if }\left|x_{n}^{q}-x_{n}\right| \leq 1\end{array}\right.$. Since $\left(x^{q}\right)_{q \geq 0}$ $\rho$-converges to $x$, then by (51), $\lim _{q \rightarrow \infty}\left|x_{n}^{q}-x_{n}\right|=0$, i.e., $\lim _{q \rightarrow \infty} x_{n}^{q}=x_{n}$. Thus, $0 \leq x_{n} \leq 1 / 2^{n}$ because $0 \leq x_{n}^{q} \leq 1 / 2^{n}$ for all $n \geq 0$.

Let the convex directed graph $G=(V(G), E(G))$ such that $V(G)=C$ and $E(G)=\left\{(x, y) \in C \times C: x_{n} \leq y_{n}, \forall n \geq 2\right\}$. The graph $G$ is transitive such that $E(G)$ is convex, $\Delta \subset E(G)$, and $G$-intervals are $\rho$-closed convex. Let $T: C \longrightarrow C$ be a mapping defined by $T\left(\left(x_{n}\right)_{n}\right)=\left(x_{n} /\left(4.2^{p^{+}}-1\right)\right)_{n}$. The mapping $T$ satisfies the condition $G-\mathscr{M}_{\gamma, \mu}$ for $\gamma=1 / 2^{p^{+}}$ $\epsilon[0,1 /(\omega(2))]$ and $\mu=1 / 2.2^{p^{+}}$. In fact, let $x$ and $y$ in $C$ such that $(x, y) \in E(G)$. It is quite easy to check that $T$ preserves edges and is $\rho$-continuous. Moreover,

$$
\begin{aligned}
(1-\gamma) \rho(x-y)+\rho(\mu(x-T y))+\rho(\mu(T x-y)) \\
\geq \sum_{n=0}^{+\infty} \frac{1}{p(n)}\left(\frac{1}{2.2^{p^{+}}}\right)^{p(n)}\left(\left|x_{n}-\frac{y_{n}}{4.2^{p^{+}}-1}\right|^{p(n)}\right. \\
\left.\quad+\left|y_{n}-\frac{x_{n}}{4.2^{p^{+}}-1}\right|^{p(n)}\right) \geq \sum_{n=0}^{+\infty} \frac{1}{p(n)}\left(\frac{1}{2.2^{p^{+}}}\right)^{p(n)} \mid\left(x_{n}-y_{n}\right) \\
\quad+\left.\frac{x_{n}-y_{n}}{4.2^{p^{+}}-1}\right|^{p(n)} \geq \sum_{n=0}^{+\infty} \frac{1}{p(n)}\left(\frac{1}{2.2^{p^{+}}}\right)^{p(n)} \\
\quad \times \frac{\left(4.2^{p^{+}}\right)^{p(n)}}{\left(4.2^{p^{+}}-1\right)^{p(n)}}\left|x_{n}-y_{n}\right|^{p(n)} \\
\geq \sum_{n=0}^{+\infty} \frac{2^{p^{-}}}{p(n)}\left|\frac{x_{n}}{4.2^{p^{+}}-1}-\frac{y_{n}}{4.2^{p^{+}}-1}\right|^{p(n)} \\
\geq \sum_{n=0}^{+\infty} \frac{1}{p(n)}\left|\frac{x_{n}}{4.2^{p^{+}}-1}-\frac{y_{n}}{4.2^{p^{+}}-1}\right|^{p(n)}=\rho(T x-T y) .
\end{aligned}
$$

For the sequel, we consider $p(n)=2+\left(2 n /\left(n^{2}+2\right)\right)$. We have $p^{-}=2$ and $p^{+}=p(1)$. Let $x^{0}=\left(1 / 2^{n}\right)_{n \geq 0} \in C$, and we have $\left(T x^{0}, x^{0}\right) \in E(G)$. Set $\alpha_{q}=\lambda \in(0, a)$, for all $q \geq 0$, where $a=\left(4.2^{p^{+}}-1\right) / 4.2^{p^{+}}$. By induction on $q$, we construct the sequence $\left(x^{q}\right)_{q \geq 0}$ given as follows: $x^{q}=$ $\left(1-\left(\lambda 4.2^{p^{+}} /\left(4.2^{p^{+}}-1\right)\right)\right)^{q} x^{0}$ for all $q \geq 0$. Moreover, we have

$$
\begin{aligned}
\rho\left(x^{q}-T x^{q}\right) & =\sum_{n \geq 0} \frac{1}{p(n)}\left|x_{n}^{q}-\frac{x_{n}^{q}}{4.2^{p^{+}}-1}\right|^{p(n)} \\
& \leq \sum_{n \geq 0} \frac{1}{p(n)}\left(1-\frac{\lambda 4.2^{p^{+}}}{4.2^{p^{+}}-1}\right)^{q p(n)}\left|x_{n}^{0}\right|^{p(n)} \\
& \leq \sum_{n \geq 0} \frac{1}{p(n)}\left(1-\frac{\lambda 4.2^{p^{+}}}{4.2^{p^{+}}-1}\right)^{q p^{+}}\left|x_{n}^{0}\right|^{p(n)} \\
& \leq\left[\left(1-\frac{\lambda 4.2^{p^{+}}}{4.2^{p^{+}}-1}\right)^{p^{+}}\right]^{q} \rho\left(x^{0}\right) \underset{p \rightarrow \infty}{\longrightarrow} 0 .
\end{aligned}
$$

Thus, $\lim _{q \rightarrow \infty} \rho\left(x^{q}-T x^{q}\right)=0$. Therefore, $T$ has a fixed point $z$ such that $\left(z, x^{0}\right) \in E(G)$.

In the sequel, we will use the fixed point set with the partial orders $\mathscr{F}_{x_{0}}^{G}(T)$ given by

$$
\mathscr{F}_{x_{0}}^{G}(T)=\left\{p \in C: T p=p \text { and }\left(x_{0}, p\right) \in E(G)\right\} .
$$

Now, we discuss the convergence of a modified version of the AK iteration scheme in order to get an accelerated iteration. The modified version is given by the following: for a 
given $x_{0} \in C$,

$$
\left\{\begin{array}{l}
x_{n+1}=T\left(\left(1-\alpha_{n}\right) y_{n}+\alpha_{n} T y_{n}\right), \\
y_{n}=T\left(\left(1-\beta_{n}\right) z_{n}+\beta_{n} T z_{n}\right), \\
z_{n}=T\left(\left(1-\delta_{n}\right) x_{n}+\delta_{n} T x_{n}\right), \quad n \in \mathbb{N},
\end{array}\right.
$$

where $\left(\alpha_{n}\right)_{n},\left(\beta_{n}\right)_{n}$, and $\left(\delta_{n}\right)_{n}$ are in $(0,1)$ such that there exists $a, b \in(0,1)$ with $a \leq \delta_{n} \leq b, C$ a convex subset of a modular space $X_{\rho}$, and $T$ satisfying the condition $G-\mathscr{M}_{\gamma, \mu}$ on $C$.

In the proof of the following lemma, we use the notations $\overline{z_{n}}=\left(1-\delta_{n}\right) x_{n}+\delta_{n} T x_{n}, \overline{y_{n}}=\left(1-\beta_{n}\right) z_{n}+\beta_{n} T z_{n}$, and $x_{n+1}^{-}=\left(1-\alpha_{n}\right) y_{n}+\alpha_{n} T y_{n}$ for all $n \geq 0$.

Lemma 19. Let $\rho$ be a convex modular and $X_{\rho}$ be a modular space endowed with a directed transitive graph, where $V(G)=C$ a nonempty $\rho$-closed convex and $\rho$-bounded, $E(G)$ is convex, and $\Delta \subseteq E(G)$. Also, let $T: C \longrightarrow C$ be a mapping preserving edges. Assume that the sequence $\left(x_{n}\right)_{n}$ is defined by iteration (55) and $\left(x_{0}, T x_{0}\right) \in E(G)$. Then, $\left(x_{n}, T x_{n}\right),\left(T x_{n}, x_{n+1}\right)$, and $\left(x_{n}, x_{n+1}\right)$ are in $E(G)$ for all $n \geq 0$.

Proof. Let $x_{0} \in C$ such that $\left(x_{0}, T x_{0}\right) \in E(G)$. We have the $G$-interval $\left[x_{0}, T x_{0}\right]=\left[x_{0}, \longrightarrow\right) \cap\left(\longleftarrow, T x_{0}\right]$ which is convex. Then, $\overline{z_{0}} \in\left[x_{0}, T x_{0}\right]$; that is, $\left(x_{0}, \overline{z_{0}}\right)$ and $\left(\overline{z_{0}}, T x_{0}\right)$ are in $E(G)$. Since $T$ preserves edges, then $\left(T x_{0}, T \bar{z}_{0}\right)$ and $\left(T \bar{z}_{0}\right.$, $\left.T^{2} x_{0}\right)$ are in $E(G)$. Thus, $\left(T x_{0}, z_{0}\right)$ and $\left(z_{0}, T^{2} x_{0}\right)$ are in $E(G)$. Since $\left(T x_{0}, z_{0}\right)$ and $\left(x_{0}, T x_{0}\right)$ are in $E(G)$, we have $\left(x_{0}, z_{0}\right) \in E(G)$ and $\left(T^{2} x_{0}, T z_{0}\right) \in E(G)$ since $T$ preserves edges. Using the transitivity of $G$ for $\left(z_{0}, T^{2} x_{0}\right)$ and $\left(T^{2}\right.$ $\left.x_{0}, T z_{0}\right)$, one finds $\left(z_{0}, T z_{0}\right) \in E(G)$. Since $\left[z_{0}, T z_{0}\right]$ is convex, then $\left(z_{0}, \bar{y}_{0}\right)$ and $\left(\bar{y}_{0}, T z_{0}\right)$ are in $E(G)$. Again, $T$ preserves edges, then $\left(T z_{0}, T \bar{y}_{0}\right)$ and $\left(T \overline{y_{0}}, T^{2} z_{0}\right)$ are in $E(G)$; that is, $\left(T z_{0}, y_{0}\right)$ and $\left(y_{0}, T^{2} z_{0}\right)$ are in $E(G)$. By transitivity of $G$ for $\left(z_{0}, T z_{0}\right)$ and $\left(T z_{0}, y_{0}\right)$, one has $\left(z_{0}, y_{0}\right) \in E(G)$. From $\left(T z_{0}, y_{0}\right)$ and since $T$ preserves edges, we get $\left(T^{2}\right.$ $\left.z_{0}, T y_{0}\right) \in E(G)$. Combining $\left(y_{0}, T^{2} z_{0}\right)$ and $\left(T^{2} z_{0}, T y_{0}\right)$, we obtain $\left(y_{0}, T y_{0}\right) \in E(G)$. Again, the $G$-interval $\left[y_{0}, T y_{0}\right]$ is convex. Then, $\left(y_{0}, \bar{x}_{1}\right)$ and $\left(\bar{x}_{1}, T y_{0}\right)$ are in $E(G)$. Since $T$ preserves edges, one has $\left(T y_{0}, T \bar{x}_{1}\right)$ and $\left(T \bar{x}_{1}, T^{2} y_{0}\right)$ which are in $E(G)$. That is, $\left(T y_{0}, x_{1}\right)$ and $\left(x_{1}, T^{2} y_{0}\right)$ are in $E(G)$. Using the transitivity of the graph $G$ for $\left(y_{0}, T y_{0}\right)$ and $\left(T y_{0}, x_{1}\right)$, one finds $\left(y_{0}, x_{1}\right) \in E(G)$. Again, $T$ preserves edges; then, $\left(T y_{0}, x_{1}\right)$ implies that $\left(T^{2} y_{0}, T x_{1}\right) \in E(G)$. Thus, combining with $\left(x_{1}, T^{2} y_{0}\right)$ and the transitivity of $G$, we get $\left(x_{1}, T x_{1}\right) \in E(G)$. Again, for the transitivity of $G$ for the edges $\left(x_{0}, z_{0}\right),\left(z_{0}, y_{0}\right)$, and $\left(y_{0}, x_{1}\right)$, one has $\left(x_{0}, x_{1}\right) \in E(G)$. Moreover, from $\left(T x_{0}, z_{0}\right),\left(z_{0}, y_{0}\right)$, and $\left(y_{0}, x_{1}\right)$, we get $\left(T x_{0}, x_{1}\right) \in E(G)$. Therefore, we have $\left(T x_{0}, x_{1}\right),\left(x_{0}, x_{1}\right)$, and $\left(x_{0}, T x_{1}\right)$ which are in $E(G)$.

Next, we assume that $\left(x_{k}, T x_{k}\right) \in E(G)$. Using the same argument as before, we deduce that $\left(x_{k}, z_{k}\right)$ and $\left(x_{k}, T z_{k}\right)$ are in $E(G)$ since $\overline{z_{k}} \in\left[x_{k}, T x_{k}\right]$. Again, $T$ preserves edges which implies that $\left(T x_{k}, z_{k}\right)$ and $\left(z_{k}, T^{2} x_{k}\right)$ are in $E(G)$. Applying transitivity of $G$ for $\left(x_{k}, T x_{k}\right)$ and $\left(T x_{k}, z_{k}\right)$, we obtain $\left(x_{k}, z_{k}\right) \in E(G)$. Since $T$ preserves edges, then from $\left(T x_{k}, z_{k}\right)$, we have $\left(T^{2} x_{k}, T z_{k}\right) \in E(G)$. Again, transitivity of $G$ implies that for $\left(z_{k}, T^{2} x_{k}\right)$ and $\left(T^{2} x_{k}, T z_{k}\right)$, we have $\left(z_{k}, T z_{k}\right) \in E(G)$.

From the convexity of $\left[z_{k}, T z_{k}\right]$, we have $\left(z_{k}, \bar{y}_{k}\right)$ and $\left(\bar{y}_{k}, T z_{k}\right)$ which are in $E(G)$. Since $T$ preserves edges, then $\left(T z_{k}, T \bar{y}_{k}\right)$ and $\left(T \bar{y}_{k}, T^{2} z_{k}\right)$ are in $E(G)$. Thus, $\left(T z_{k}, y_{k}\right)$ and $\left(y_{k}, T^{2} z_{k}\right)$ are in $E(G)$. Applying transitivity of $G$ for $\left(z_{k}, T z_{k}\right)$ and $\left(T z_{k}, y_{k}\right)$, we get $\left(z_{k}, y_{k}\right) \in E(G)$. Since $T$ preserves edges, then $\left(T^{2} z_{k}, T y_{k}\right) \in E(G)$. Again, by transitivity for $\left(y_{k}, T^{2} z_{k}\right)$ and $\left(T^{2} z_{k}, T y_{k}\right)$, one has $\left(y_{k}, T y_{k}\right) \in E(G)$. Since $\left[y_{k}, T y_{k}\right]$ is convex, then $x_{k+1}^{-} \in\left[y_{k}, T y_{k}\right]$; that is, $\left(y_{k}, x_{k+1}^{-}\right)$and $\left(x_{k+1}^{-}, T y_{k}\right)$ are in $E(G)$. Since the mapping $T$ preserves edges, then $\left(T y_{k}, T x_{k+1}^{-}\right)$and $\left(T x_{k+1}^{-}, T^{2} y_{k}\right)$ are in $E(G)$; that is, $\left(T y_{k}, x_{k+1}\right)$ and $\left(x_{k+1}, T^{2} y_{k}\right)$ are in $E(G)$. Again, the transitivity of $G$ for $\left(y_{k}, T y_{k}\right)$ and $\left(T y_{k}, x_{k+1}\right)$ implies that $\left(y_{k}, x_{k+1}\right) \in E(G)$.

Since $T$ preserves edges, then $\left(T y_{k}, x_{k+1}\right)$ implies that $\left(T^{2} y_{k}, T x_{k+1}\right) \in E(G)$. Transitivity of $G$ for $\left(x_{k+1}, T^{2} y_{k}\right)$ and $\left(T^{2} y_{k}, T x_{k+1}\right)$ leads to $\left(x_{k+1}, T x_{k+1}\right) \in E(G)$. Moreover, from $\left(x_{k}, z_{k}\right),\left(z_{k}, y_{k}\right)$, and $\left(y_{k}, x_{k+1}\right)$, we deduce that $\left(x_{k}\right.$, $\left.x_{k+1}\right) \in E(G)$. And from $\left(T x_{k}, z_{k}\right),\left(z_{k}, y_{k}\right)$, and $\left(y_{k}, x_{k+1}\right)$, one finds $\left(T x_{k}, x_{k+1}\right) \in E(G)$. By $\left(x_{k}, T x_{k}\right)$ and $\left(x_{k}, x_{k+1}\right)$ and since $T$ preserves edges, we obtain $\left(x_{k}, T x_{k+1}\right) \in E(G)$.

Therefore, $\left(x_{k}, x_{k+1}\right),\left(x_{k+1}, T x_{k+1}\right),\left(T x_{k}, x_{k+1}\right)$, and $\left(x_{k}\right.$, $\left.T x_{k+1}\right)$ are in $E(G)$. Finally, we conclude, by induction, that $\left(x_{k}, T x_{k}\right),\left(x_{k}, x_{k+1}\right)$, and $\left(T x_{k}, x_{k+1}\right)$ are in $E(G)$ for all $k \geq 0$.

Lemma 20. Let $\rho$ be a convex modular and $X_{\rho}$ be a modular space endowed with a directed transitive graph $G=(V(G), E$ $(G)$ ), where $V(G)=C$ is a nonempty $\rho$-closed convex subset, $E(G)$ is convex, and $\Delta \subset E(G)$. Let $T: C \longrightarrow C$ be a mapping satisfying $G-\mathscr{M}_{\gamma, \mu}$ on $C$. Assume that there exists $x_{0} \in C$ such that $\left(x_{0}, T x_{0}\right) \in E(G)$ and $\mathscr{F}_{x_{0}}^{G}(T)$ is nonempty. Then, $\lim _{n} \rho$ $\left(x_{n}-p\right)$ exists for all $p \in \mathscr{F}_{x_{0}}^{G}(T)$, where $\left(x_{n}\right)_{n}$ is defined by (55).

Proof. Assume that there exists $x_{0} \in C$ such that $\left(x_{0}, T x_{0}\right) \in$ $E(G)$ and $\mathscr{F}_{x_{0}}^{G}(T)$ is nonempty. Fix $p \in \mathscr{F}_{x_{0}}^{G}(T)$. Using the convexity of $E(G)$, one can prove by induction that $\left(x_{n}, p\right)$, $\left(y_{n}, p\right)$, and $\left(z_{n}, p\right)$ are in $E(G)$ for all $n \geq 0$.

Moreover, we have

$$
\begin{aligned}
\rho\left(x_{n+1}-p\right) & =\rho\left(T\left(\left(1-\alpha_{n}\right) y_{n}+\alpha_{n} T y_{n}\right)-p\right) \\
& \leq\left(1-\alpha_{n}\right) \rho\left(y_{n}-p\right)+\alpha_{n} \rho\left(T y_{n}-p\right) \\
& \leq \rho\left(y_{n}-p\right) \leq \rho\left(T\left(\left(1-\beta_{n}\right) z_{n}+\beta_{n} T z_{n}\right)-p\right) \\
& \leq \rho\left(z_{n}-p\right) \leq \rho\left(T\left(\left(1-\delta_{n}\right) x_{n}+\delta_{n} T x_{n}\right)-p\right) \\
& \leq \rho\left(x_{n}-p\right),
\end{aligned}
$$

for all $n \geq 1$. Hence, $\left(\rho\left(x_{n}-p\right)\right)_{n}$ is a decreasing sequence and bounded in $\mathbb{R}_{+}$. Thus, $\lim _{n} \rho\left(x_{n}-p\right)$ exists. 
Theorem 21. Let $\rho$ be a convex modular (UUC1) satisfying $\Delta_{2}$-type condition and $X_{\rho}$ be a modular space endowed with a directed transitive graph $G=(V(G), E(G))$, where $V(G)=C$ is a nonempty $\rho$-closed convex subset, $E(G)$ is convex, and $\Delta \subset E(G)$. Let $T: C \longrightarrow C$ be a mapping satisfying $G-\mathscr{M}_{\gamma, \mu}$ on $C$. Assume that there exists $x_{0} \in C$ such that $\left(x_{0}, T x_{0}\right) \in E(G)$ and $\mathscr{F}_{x_{0}}^{G}(T)$ is nonempty. Then, $\lim _{n} \rho\left(x_{n}-T x_{n}\right)=0$.

Proof. Let $x_{0} \in C$ such that $\left(x_{0}, T x_{0}\right) \in E(G)$ and $\mathscr{F}_{x_{0}}^{G}(T)$ is nonempty. Fix $p \in \mathscr{F}_{x_{0}}^{G}(T)$. By Lemma $20, \lim _{n} \rho\left(x_{n}-p\right)=r$ $\geq 0$ exists. We assume that $r>0$, because if $r=0$, there is nothing to prove. We have

$$
\limsup _{n \rightarrow \infty} \rho\left(T x_{n}-p\right) \leq \limsup _{n \rightarrow \infty} \rho\left(x_{n}-p\right)=r .
$$

Otherwise, by the inequalities (56), we have

$$
\rho\left(x_{n+1}-p\right) \leq \rho\left(\left(1-\delta_{n}\right)\left(x_{n}-p\right)+\delta_{n}\left(T x_{n}-p\right)\right) \leq \rho\left(x_{n}-p\right),
$$

for all $n \geq 1$. Hence,

$$
\lim _{n} \rho\left(\delta_{n}\left(T x_{n}-p\right)+\left(1-\delta_{n}\right)\left(x_{n}-p\right)\right)=r .
$$

By Lemma 10 and Equations (57) and (59), we obtain $\lim _{n} \rho\left(x_{n}-T x_{n}\right)=0$.

Theorem 22. Let $\rho$ be a convex modular (UUC1) satisfying $\Delta_{2}$-type condition and $X_{\rho}$ be a modular space endowed with a directed transitive graph $G=(V(G), E(G))$, where $V(G)=C$ is a nonempty $\rho$-sequentially compact convex and $\rho$-bounded subset having property $(P), E(G)$ is convex, and $\Delta \subset E(G)$. Also, let $T: C \longrightarrow C$ be a mapping satisfying $G-M_{\gamma, \mu}$ on $C$ with $\gamma \in[0,1 /(\omega(2))]$. Assume that there exists $x_{0} \in C$ such that $\left(x_{0}, T x_{0}\right) \in E(G)$ and $\mathscr{F}_{x_{0}}^{G}(T)$ is nonempty. Then, the sequence $\left(x_{n}\right)_{n}$ given by (55) $\rho$-converges to a fixed point of $T$.

Proof. Assume that there exists $x_{0} \in C$ such that $\left(x_{0}, T x_{0}\right) \in$ $E(G)$. Since $C$ is $\rho$-sequentially compact, then $\left(x_{n}\right)_{n}$ has a subsequence $\left(x_{\varphi(n)}\right)_{n}$ which $\rho$-converges to $z \in C$, where $\varphi$ is an increasing function on $\mathbb{N}$. Moreover, $\left(x_{\varphi(n)}, x_{\varphi(n+1)}\right) \in$ $E(G)$ for all $n \geq 0$. Thus, the property $(P)$ implies that $\left(x_{\varphi(n)}, z\right) \in E(G)$ for all $n \geq 0$. Next, let us prove that $z$ is a fixed point of $T$.

Case 1. If there exists a subsequence $\left(x_{\varphi \circ \sigma_{1}(n)}\right)_{n}$ such that $\gamma \rho$ $\left(x_{\varphi \circ \sigma_{1}(n)}-T x_{\varphi \circ \sigma_{1}(n)}\right) \leq \rho\left(x_{\varphi \circ \sigma_{1}(n)}-z\right)$ for all $n \geq 0$, that is, $T$ satisfies (ii, a) of Proposition 17 for the subsequence $\left(x_{\varphi \circ \sigma_{1}(n)}\right)_{n}$ and $c=\gamma \omega(2)$. By the condition $G-\mathscr{M}_{\gamma, \mu}$, we obtain

$$
\begin{aligned}
& \rho\left(T x_{\varphi \circ \sigma_{1}(n)}-T z\right) \leq(1-\gamma) \rho\left(x_{\varphi \circ \sigma_{1}(n)}-z\right) \\
& \quad+\rho\left(\mu\left(x_{\varphi \circ \sigma_{1}(n)}-T z\right)\right)+\rho\left(\mu\left(z-T x_{\varphi \circ \sigma_{1}(n)}\right)\right) .
\end{aligned}
$$

For all $n \geq 0$,

$$
\begin{aligned}
\rho\left(T x_{\varphi \circ \sigma_{1}(n)}-T z\right) \leq & (1-\gamma) \rho\left(x_{\varphi \circ \sigma_{1}(n)}-z\right) \\
& +\mu\left(\rho\left(x_{\varphi \circ \sigma_{1}(n)}-T x_{\varphi \circ \sigma_{1}(n)}\right)\right. \\
& \left.+\rho\left(T x_{\varphi \circ \sigma_{1}(n)}-T z\right)\right) \\
& +\mu\left(\rho\left(x_{\varphi \circ \sigma_{1}(n)}-T x_{\varphi \circ \sigma_{1}(n)}\right)\right. \\
& \left.+\rho\left(x_{\varphi \circ \sigma_{1}(n)}-z\right)\right) \\
\leq & (1-\gamma+\mu) \rho\left(x_{\varphi \circ \sigma_{1}(n)}-z\right) \\
& +2 \mu \rho\left(x_{\varphi \circ \sigma_{1}(n)}-T x_{\varphi \circ \sigma_{1}(n)}\right) \\
& +\mu \rho\left(T x_{\varphi \circ \sigma_{1}(n)}-T z\right) .
\end{aligned}
$$

Hence, $\quad(1-\mu) \rho\left(T x_{\varphi \circ \sigma_{1}(n)}-T z\right) \leq(1-\gamma+\mu) \rho\left(x_{\varphi \circ \sigma_{1}(n)}\right.$ $-z)+2 \mu \rho\left(x_{\varphi \circ \sigma_{1}(n)}-T x_{\varphi \circ \sigma_{1}(n)}\right)$ for all $n \geq 0$. It follows

$$
\begin{gathered}
\rho\left(T x_{\varphi \circ \sigma_{1}(n)}-T z\right) \leq \rho\left(x_{\varphi \circ \sigma_{1}(n)}-z\right) \\
+\frac{2 \mu}{1-\mu} \rho\left(x_{\varphi \circ \sigma_{1}(n)}-T x_{\varphi \circ \sigma_{1}(n)}\right),
\end{gathered}
$$

since $2 \mu \leq \gamma$. Taking $\lim _{n \rightarrow \infty}$, we obtain $\lim _{n} \rho\left(T x_{\varphi \circ \sigma_{1}(n)}\right.$ $-T z)=0$. Otherwise,

$$
\begin{aligned}
\rho\left(x_{\varphi \circ \sigma_{1}(n)}-T z\right) \leq & \frac{\omega(2)}{2} \rho\left(x_{\varphi \circ \sigma_{1}(n)}-T x_{\varphi \circ \sigma_{1}(n)}\right) \\
& +\frac{\omega(2)}{2} \rho\left(T x_{\varphi \circ \sigma_{1}(n)}-T z\right),
\end{aligned}
$$

for all $n \geq 1$. Therefore, again by $\lim _{n \rightarrow \infty}$ and by Theorem 21 , $\lim _{n} \rho\left(x_{\varphi \circ \sigma_{1}(n)}-T z\right)=0$.

Case 2. If there exists a subsequence $\left(x_{\varphi \circ \sigma_{2}(n)}\right)_{n}$ of $\left(x_{\varphi(n)}\right)_{n}$ such that $\gamma \rho\left(T^{2} x_{\varphi \circ \sigma_{2}(n)}-T x_{\varphi \circ \sigma_{2}(n)}\right) \leq \rho\left(T x_{\varphi \circ \sigma_{2}(n)}-z\right)$ for all $n \geq 0$, that is, $T$ satisfies (ii, b) of Proposition 17 for the subsequence $\left(x_{\varphi \circ \sigma_{2}(n)}\right)_{n}$ and $c=\gamma \omega(2)$. By the condition $\mathscr{M}_{\gamma, \mu}$, we obtain

$$
\begin{aligned}
& \rho\left(T^{2} x_{\varphi \circ \sigma_{2}(n)}-T z\right) \leq(1-\gamma) \rho\left(T x_{\varphi \circ \sigma_{2}(n)}-z\right) \\
& \quad+\rho\left(\mu\left(T x_{\varphi \circ \sigma_{2}(n)}-T z\right)\right)+\rho\left(\mu\left(T^{2} x_{\varphi \circ \sigma_{2}(n)}-z\right)\right) .
\end{aligned}
$$


Hence,

$$
\begin{aligned}
& \rho\left(T^{2} x_{\varphi \circ \sigma_{2}(n)}-T z\right) \leq(1-\gamma) \rho\left(T x_{\varphi \circ \sigma_{2}(n)}-z\right) \\
& \quad+\mu\left(\rho\left(T x_{\varphi \circ \sigma_{2}(n)}-T^{2} x_{\varphi \circ \sigma_{2}(n)}\right)+\rho\left(T^{2} x_{\varphi \circ \sigma_{2}(n)}-T z\right)\right) \\
& \quad+\mu\left(\rho\left(T^{2} x_{\varphi \circ \sigma_{2}(n)}-T x_{\varphi \circ \sigma_{2}(n)}\right)+\rho\left(T x_{\varphi \circ \sigma_{2}(n)}-z\right)\right),
\end{aligned}
$$

for all $n \geq 0$. This implies $(1-\mu) \rho\left(T^{2} x_{\varphi \circ \sigma_{2}(n)}-T z\right) \leq(1-\gamma$ $+\mu) \rho\left(T x_{\varphi \circ \sigma_{2}(n)}-z\right)+2 \mu \rho\left(T x_{\varphi \circ \sigma_{2}(n)}-T^{2} x_{\varphi \circ \sigma_{2}(n)}\right)$ for all $n$ $\geq 0$. Hence,

$$
\begin{aligned}
\rho( & \left.T^{2} x_{\varphi \circ \sigma_{2}(n)}-T z\right) \leq \rho\left(T x_{\varphi \circ \sigma_{2}(n)}-z\right) \\
& +\frac{2 \mu}{1-\mu} \rho\left(T x_{\varphi \circ \sigma_{2}(n)}-T^{2} x_{\varphi \circ \sigma_{2}(n)}\right) \\
\leq & \frac{\omega(2)}{2} \rho\left(T x_{\varphi \circ \sigma_{2}(n)}-x_{\varphi \circ \sigma_{2}(n)}\right)+\frac{\omega(2)}{2} \rho\left(x_{\varphi \circ \sigma_{2}(n)}-z\right) \\
& +\frac{2 \mu}{1-\mu} \rho\left(x_{\varphi \circ \sigma_{2}(n)}-T x_{\varphi \circ \sigma_{2}(n)}\right),
\end{aligned}
$$

since $2 \mu \leq \gamma$ for all $n \geq 1$. Taking the limit as $n$ goes to infinity, we get $\lim _{n} \rho\left(T^{2} x_{\varphi \circ \sigma_{2}(n)}-T z\right)=0$. Moreover, for all $n \geq 0$, we have

$$
\begin{aligned}
\rho\left(x_{\varphi \circ \sigma_{2}(n)}-T z\right) \leq & \frac{\omega(2)}{2} \rho\left(x_{\varphi \circ \sigma_{2}(n)}-T x_{\varphi \circ \sigma_{2}(n)}\right) \\
& +\frac{\omega(2)}{2} \rho\left(T x_{\varphi \circ \sigma_{2}(n)}-T z\right) \\
\leq & \frac{\omega(2)}{2} \rho\left(x_{\varphi \circ \sigma_{2}(n)}-T x_{\varphi \circ \sigma_{2}(n)}\right) \\
& +\frac{\omega(2)^{2}}{4} \rho\left(T x_{\varphi \circ \sigma_{2}(n)}-T^{2} x_{\varphi \circ \sigma_{2}(n)}\right) \\
& +\frac{\omega(2)^{2}}{4} \rho\left(T^{2} x_{\varphi \circ \sigma_{2}(n)}-T z\right) \\
\leq & \frac{\omega(2)}{2}\left(1+\frac{\omega(2)}{2}\right) \rho\left(x_{\varphi \circ \sigma_{2}(n)}-T x_{\varphi \circ \sigma_{2}(n)}\right) \\
& +\frac{\omega(2)^{2}}{4} \rho\left(T^{2} x_{\varphi \circ \sigma_{2}(n)}-T z\right) .
\end{aligned}
$$

By $\lim _{n \rightarrow \infty}$, we get $\lim _{n} \rho\left(x_{\varphi \circ \sigma_{2}(n)}-T z\right)=0$. Thus, from both cases, we conclude that $T z=z$, since $\left(x_{\varphi(n)}\right)_{n} \rho$-converges to $z$. Hence, $z$ is a fixed point of the mapping $T$.

In order to complete the proof, let us show that $\left(x_{n}\right)_{n}$ $\rho$-converges to $z$. By Lemma 20, $\lim _{n} \rho\left(x_{n}-p\right)$ exists, for all $p \in \mathscr{F}_{x_{1}}^{ \pm}(T)$. Since $\left(x_{n}\right)_{n}$ is a $G$-decreasing sequence, then $\left(x_{0}, x_{\varphi(n)}\right) \in E(G)$ for all $n \geq 0$. Moreover, we have $\left(x_{\varphi(n)}, z\right)$ $\in E(G)$ for all $n \geq 0$. Thus, $z \in \mathscr{F}_{x_{0}}^{G}(T)$. Hence, $\lim _{n} \rho\left(x_{n}-z\right)$ exists. Since $\left(x_{\varphi(n)}\right)_{n} \rho$-converges to $z$, then $\lim _{n} \rho\left(x_{\varphi(n)}-z\right)=0$. Thus, $\lim _{n} \rho\left(x_{n}-z\right)=0$. Therefore, $\left(x_{n}\right)_{n} \rho$-converges to $z$ a fixed point of $T$.

\section{Numerical Example}

Example 2. Let $X_{\rho}=\mathbb{R}$, where the modular $\rho$ is defined by $\rho$ $(x)=|x|$, for all $x \in X_{\rho}$, with $\omega(2)=2$, and let the graph $G$ $=(V(G), E(G))$ such that $V(G)=[-1,1]$ and $E(G)=\{(x, y)$ $\in[-1,1] \times[-1,1]: x \leq y$ and $|x-y| \leq 1\}$. Consider the mapping $T:[-1,1] \longrightarrow[-1,1]$ defined by

$$
T x= \begin{cases}x+\frac{2}{3} & \text { if }-1 \leq x \leq \frac{-1}{2} \\ \frac{x+1}{3} & \text { if } \frac{-1}{2} \leq x \leq 1\end{cases}
$$

The mapping $T$ preserves edges and satisfies the condition $\mathscr{M}_{\gamma, \mu}$ for $\gamma=1 / 2$ and $\mu=1 / 4$. Indeed, if $x$ and $y$ are in $[-1,-1 / 2]$ or in $[-1 / 2,1]$ such that $(x, y) \in E(G)$, we have $T$ which is $\rho$-nonexpansive and preserves edges. Then, it is $G-\mathscr{M}_{\gamma, \mu}$. Otherwise, if $x \in[-1,-1 / 2]$ and $y \in[$ $-1 / 2,1]$, then $(x, y) \in E(G)$, and we have $\rho(T x-T y)=(1 /$ 3) $y-x-(1 / 3)$. Otherwise,

$$
\begin{aligned}
& (1-\gamma) \rho(x-y)+\rho(\mu(x-T y))+\rho(\mu(y-T x)) \\
& \quad \geq\left(1-\frac{1}{2}\right)(y-x)+\frac{1}{4}\left(1-\frac{2 y}{3}\right)=\frac{1}{3} y-\frac{1}{2} x+\frac{1}{4} \\
& \quad \geq \frac{1}{3} y-x-\frac{1}{3} .
\end{aligned}
$$

If $x_{1}=1$, we have $\left(T x_{1}, x_{1}\right) \in E(G)$ and all the hypotheses of Theorem 18 are fulfilled. Then, the mapping $T$ has a fixed point $x^{*}=1 / 2$.

To illustrate the convergence of the proposed algorithm, we provide some numerical results and a comparison with other recent iterations: AK iteration [24], M iteration [34], K iteration [35], and the iteration of Piri [22]. Firstly, we show the convergence behavior of the iterative process (55) with different initial points. To do this, we take $\alpha_{n}=$ $((3 n+8) /(9 n+12))^{8}, \quad \beta_{n}=((n+4) /(4 n+5)), \quad \delta_{n}=$ $\sqrt{2 n /(3(n+5))}$, and $\rho\left(x_{n}-x^{*}\right)<10^{-15}$ as a stop criterion. From the given initial points $x_{1}^{(1)}=-1, x_{1}^{(2)}=-0.5, x_{1}^{(3)}=0$, and $x_{1}^{(4)}=1$, the convergence behaviors of algorithm (55) are displayed in Table 1 and graphicaly in Figures 1 and 2 . The numerical results of Table 1 show that the decrease of the initial point $x_{1}$ affects the convergence speed. That is, the sequence $\left(x_{n}\right)_{n}$ generated by algorithm (55) will converge in less number of iterations to a fixed point when $x_{1}$ is decreased.

We show, in Table 2, the stability of the iterative process (55) based on different iteration parameters $\alpha_{n}, \beta_{n}$, and $\delta_{n}$. Moreover, for more precision of the convergence to the fixed point $x^{*}$, we take also $\rho\left(x_{n}-x^{*}\right)<10^{-15}$ as a stop criterion. Table 2 shows that different parameters $\alpha_{n}, \beta_{n}$, and $\delta_{n}$ which have a small effect on the number of iterations needed to achieve the fixed point $x^{*}$. Finally, we compare the iteration numbers of the proposed scheme 
TABLE 1: Influence of initial points.

\begin{tabular}{lcccc}
\hline Iter. $(n)$ & $x_{1}^{(1)}$ & $x_{1}^{(2)}$ & $x_{1}^{(3)}$ & $x_{1}^{(4)}$ \\
\hline 1 & -1 & -0.5 & 0 & 1 \\
2 & 0.457408902872947 & 0.481931049703675 & 0.490965524851837 & 0.509034475148163 \\
3 & 0.499226830094190 & 0.499671988524808 & 0.499835994262404 & 0.500164005737596 \\
4 & 0.499986161980696 & 0.499994129325144 & 0.499997064662572 & 0.500002935337428 \\
5 & 0.499999756543944 & 0.499999896715612 & 0.499999948357806 & 0.500000051642194 \\
6 & 0.499999995786796 & 0.499999998212580 & 0.499999999106290 & 0.500000000893710 \\
7 & 0.499999999928167 & 0.499999999969525 & 0.499999999984763 & 0.500000000015237 \\
8 & 0.499999999998791 & 0.499999999999487 & 0.499999999999744 & 0.500000000000256 \\
9 & 0.499999999999980 & 0.499999999999992 & 0.499999999999996 & 0.500000000000004 \\
10 & 0.5 & 0.5 & 0.5 & 0.5 \\
\hline
\end{tabular}
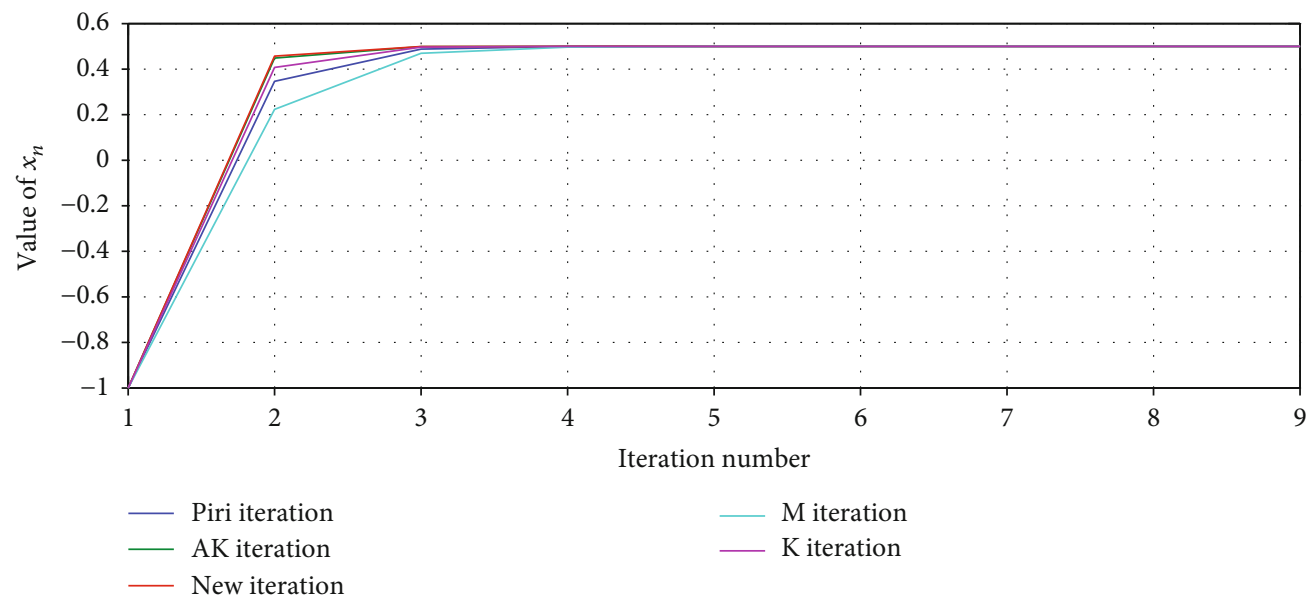

FIGURE 1: Influence of initial points $x_{1}{ }^{(1)}=-1$.

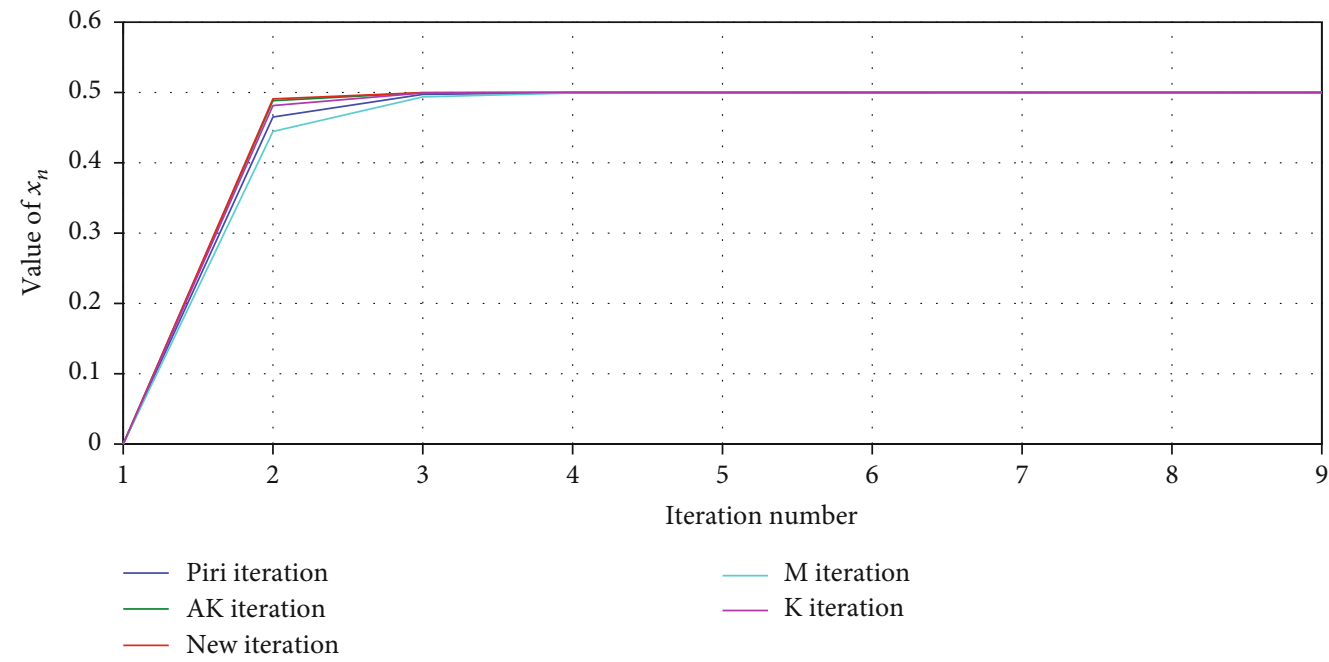

FIGURE 2: Influence of initial points $x_{1}{ }^{(3)}=0$.

(55) with the other processes. The numerical results of Table 2 prove that our scheme is advantageous because it required less number of iterations for the convergence than the other schemes.

\section{Conclusions}

Throughout the paper, we have established an existence result and some convergence theorems of a new iterative 
TABLE 2: Influence of parameters and initial points.

\begin{tabular}{|c|c|c|c|c|c|}
\hline Param.-init. points & $\begin{array}{l}\text { AK iter. } \\
\text { [24] }\end{array}$ & $\begin{array}{l}\text { M iter. } \\
\text { [34] }\end{array}$ & $\begin{array}{l}\text { K iter. } \\
{[35]}\end{array}$ & $\begin{array}{l}\text { Piri } \\
{[22]}\end{array}$ & $\begin{array}{l}\text { Iter. } \\
(55)\end{array}$ \\
\hline \multicolumn{6}{|c|}{ Param. 1: $\alpha_{n}=0.7, \beta_{n}=0.2, \delta_{n}=0.9$} \\
\hline-1 & 9 & 13 & 11 & 13 & 8 \\
\hline-0.5 & 9 & 13 & 11 & 12 & 8 \\
\hline 0 & 9 & 13 & 11 & 12 & 7 \\
\hline 1 & 9 & 13 & 11 & 12 & 7 \\
\hline \multicolumn{6}{|c|}{ Param. 2: $\alpha_{n}=(3 n /(8 n+4))^{2}, \beta_{n}=n /(4 n+2), \delta_{n}=\sqrt{2 n /(3 n+5)}$} \\
\hline-1 & 11 & 16 & 11 & 15 & 9 \\
\hline-0.5 & 10 & 16 & 11 & 15 & 9 \\
\hline 0 & 10 & 16 & 11 & 15 & 9 \\
\hline 1 & 10 & 16 & 11 & 15 & 9 \\
\hline \multicolumn{6}{|c|}{$\begin{array}{l}\text { Param. 3: } \alpha_{n}=\left(4 n^{2}+n^{3}\right) /\left(2 n^{3}+10\right), \beta_{n}=n^{2} /(2 n+6)^{2}, \delta_{n}=1- \\
((6 n+2) /(n+1))\end{array}$} \\
\hline-1 & 9 & 13 & 11 & 12 & 8 \\
\hline-0.5 & 9 & 12 & 11 & 12 & 8 \\
\hline 0 & 9 & 12 & 11 & 12 & 7 \\
\hline 1 & 9 & 12 & 11 & 12 & 8 \\
\hline
\end{tabular}

scheme for the class of mappings satisfying the condition $G-\mathscr{M}_{\gamma, \mu}$ in the framework of modular spaces endowed with a directed graph $G$. Further, we gave an example of this class of mapping to space $\ell_{p(\cdot)}$ endowed with a directed graph $G$. Our results are generalization of several results as in relevant items from the reference section of this paper, as well as in the literature in general.

\section{Abbreviations}

UCi: Uniform convexity

UUCi: Unique uniform convexity

UCED: Uniform convexity in every direction

UUCED: Unique uniform convexity in every direction

SC: $\quad$ Strict convexity.

\section{Data Availability}

No data were used to support this study.

\section{Conflicts of Interest}

The authors declare that they have no conflicts of interest regarding the publication of this paper.

\section{References}

[1] H. Nakano, Modulared Semi-Ordered Linear Spaces, Maruzen Co, 1st edition, 1950, https://www.worldcat.org/title/ modulared-semi-ordered-linear-spaces/oclc/36642.

[2] J. Musielak, Orlicz Spaces and Modular Spaces, Lecture Notes in Mathematics, Springer-Verlag, Berlin Heidelberg, 1983.

[3] A. C. M. Ran and M. C. B. Reurings, "A fixed point theorem in partially ordered sets and some applications to matrix equa- tions," Proceedings of the American Mathematical Society, vol. 132, no. 5, pp. 1435-1444, 2004.

[4] J. J. Nieto and R. Rodríguez-López, "Contractive mapping theorems in partially ordered sets and applications to ordinary differential equations," Order, vol. 22, no. 3, pp. 223-239, 2005.

[5] Z. Drici, F. A. McRae, and J. Vasundhara Devi, "Fixed point theorems for mixed monotone operators with PPF dependence," Nonlinear Analysis: Theory, Methods \& Applications, vol. 69, no. 2, pp. 632-636, 2008.

[6] J. Harjani and K. Sadarangani, "Generalized contractions in partially ordered metric spaces and applications to ordinary differential equations," Nonlinear Analysis, vol. 72, no. 3-4, pp. 1188-1197, 2010.

[7] A. Petrusel and I. A. Rus, "Fixed point theorems in ordered Lspaces," Proceedings of American Mathematical Society, vol. 134, pp. 411-418, 2006.

[8] J. Jachymski, "The contraction principle for mappings on a metric space with a graph," Proceedings of the American Mathematical Society, vol. 136, no. 4, pp. 1359-1373, 2008.

[9] M. R. Alfuraidan, "Remarks on Caristi's fixed point theorem in metric spaces with a graph," Fixed Point Theory and Applications, vol. 2014, no. 1, 2014.

[10] M. R. Alfuraidan, "On monotone Ćirić quasi-contraction mappings with a graph," Fixed Point Theory and Applications, vol. 2015, no. 1, 2015.

[11] I. Beg, A. R. Butt, and S. Radojević, "The contraction principle for set valued mappings on a metric space with a graph," Computers \& Mathematics with Applications, vol. 60, no. 5, pp. 1214-1219, 2010.

[12] K. Fallahi and G. Soleimani Rad, "Fixed points of a $\varphi$-G-contractive mapping with respect to a $c$-distance on an abstract metric space endowed with a graph," Mathematical Notes, vol. 105, no. 5-6, pp. 781-788, 2019.

[13] K. Fallahi, A. Petrusel, and G. S. Rad, "Fixed point results for pointwise Chatterjea type mappings with respect to a 
c-distance in cone metric spaces endowed with a graph," UPB Scientific Bulletin, (Series A), vol. 80, no. 1, pp. 4754, 2018.

[14] M. Öztürk, M. Abbas, and E. Girgin, "Fixed points of mappings satisfying contractive condition of integral type in modular spaces endowed with a graph," Fixed Point Theory and Applications, vol. 2014, no. 1, 2014.

[15] M. R. Alfuraidan, "The contraction principle for mappings on a modular metric space with a graph," Fixed Point Theory and Applications, vol. 2015, no. 1, 2015.

[16] M. R. Alfuraidan, "Fixed points of multivalued mappings in modular function spaces with a graph," Fixed Point Theory and Applications, vol. 2015, no. 1, 2015.

[17] M. Abbas and T. Nazir, "A new faster iteration process applied to constrained minimization and feasibility problems," Matematicki Vesnik, vol. 66, pp. 223-234, 2014.

[18] R. P. Agarwal, D. O’Regan, and D. R. Sahu, "Iterative construction of fixed points of nearly asymptotically nonexpansive mappings," Journal of Nonlinear and Convex Analysis, vol. 8, no. 1, pp. 61-79, 2007.

[19] S. Ishikawa, "Fixed points by a new iteration method," Proceedings of the American Mathematical Society, vol. 44, no. 1, p. 147, 1974.

[20] W. R. Mann, "Mean value methods in iteration," Proceedings of the American Mathematical Society, vol. 4, no. 3, pp. 506$510,1953$.

[21] M. A. Noor, "New approximation schemes for general variational inequalities," Journal of Mathematical Analysis and Applications, vol. 251, no. 1, pp. 217-229, 2000.

[22] H. Piri, B. Daraby, S. Rahrovi, and M. Ghasemi, "Approximating fixed points of generalized $\alpha$-nonexpansive mappings in Banach spaces by new faster iteration process," Numerical Algorithms, vol. 81, no. 3, pp. 1129-1148, 2019.

[23] B. S. Thakur, D. Thakur, and M. Postolache, "A new iterative scheme for numerical reckoning fixed points of Suzuki's generalized nonexpansive mappings," Applied Mathematics and Computation, vol. 275, pp. 147-155, 2016.

[24] K. Ullah and M. Arshad, "On different results for new three step iteration process in Banach spaces," SpringerPlus, vol. 5, no. 1, p. 1616, 2016.

[25] S. M. A. Aleomraninejad, S. Rezapour, and N. Shahzad, "Some fixed point results on a metric space with a graph," Topology and its Applications, vol. 159, no. 3, pp. 659-663, 2012.

[26] A. A. N. Abdou and M. A. Khamsi, "Fixed point theorems in modular vector spaces," The Journal of Nonlinear Sciences and Applications, vol. 10, no. 8, pp. 4046-4057, 2017.

[27] P. Kumam, "Fixed point theorems for nonexpansive mappings in modular spaces," Archivum Mathematicum, vol. 40, no. 4, pp. 345-353, 2004.

[28] M. Alfuraidan, M. A. Khamsi, and N. Manav, "A fixed point theorem for uniformly Lipschitzian mappings in modular vector spaces," Filomat, vol. 31, no. 17, pp. 5435-5444, 2017.

[29] B. Dehaish and M. Khamsi, "Fibonacci-Mann iteration for monotone asymptotically nonexpansive mappings in modular spaces," Symmetry, vol. 10, no. 10, p. 481, 2018.

[30] A. Bejenaru and M. Postolache, "On Suzuki mappings in modular spaces,” Symmetry, vol. 11, no. 3, p. 319, 2019.

[31] U. S. R. Murty and A. Bondy, Graph Theory (Graduate Texts in Mathematics 244), Springer-Verlag London, 2008.
[32] M. Bachar, M. Bounkhel, and M. A. Khamsi, "Uniform convexity in $\ell_{\mathrm{p}(\cdot),}$, Journal of Nonlinear Sciences and Applications, vol. 10, no. 10, pp. 5292-5299, 2017.

[33] M. A. Khamsi and W. M. Kozlowski, Fixed Point Theory in Modular Function Spaces, With a foreword by W. A. Kirk, Birkhauser/Springer, Cham, 2015.

[34] K. Ullah and M. Arshad, "Numerical reckoning fixed points for Suzuki's generalized nonexpansive mappings via new iteration process," Univerzitet $u$ Nišu, vol. 32, no. 1, pp. 187-196, 2018.

[35] N. Hussain, K. Ullah, and M. Arshad, "Fixed point approximation of Suzuki generalized nonexpansive mapping via new faster iteration process," Journal of Nonlinear and Convex Analysis, vol. 19, no. 8, 2018, https://arxiv.org/abs/1802.09888. 